https://helda.helsinki.fi

\title{
Novel anti-invasive properties of a Fascin1 inhibitor on colorectal cancer cells
}

\section{Montoro-Garcia, Silvia}

2020-03

Montoro-Garcia , S , Alburquerque-Gonzalez , B , Bernabe-Garcia , A , Bernabe-Garcia , M , Rodrigues , P C , den-Haan , H, Luque , I, Jose Nicolas , F , Perez-Sanchez , H, Luisa

Cayuela, M , Salo, T \& Conesa-Zamora , P 2020 , ' Novel anti-invasive properties of a Fascin1 inhibitor on colorectal cancer cells ', Journal of Molecular Medicine , vol. 98 , no. 3 , pp. 383-394 . https://doi.org/10.1007/s00109-020-01877-z

http://hdl.handle.net/10138/325423

https://doi.org/10.1007/s00109-020-01877-z

acceptedVersion

Downloaded from Helda, University of Helsinki institutional repository.

This is an electronic reprint of the original article.

This reprint may differ from the original in pagination and typographic detail.

Please cite the original version. 


\section{Journal of Molecular Medicine \\ Novel anti-invasive properties of a Fascin1 inhibitor on colorectal cancer cells --Manuscript Draft--}

Manuscript Number:

Full Title:

Article Type:

Corresponding Author:
JMME-D-19-00045R2

Novel anti-invasive properties of a Fascin1 inhibitor on colorectal cancer cells

Original Article

Pablo Conesa-Zamora, Ph.D, Pharm.D

Santa Lucia University Hospital

Cartagena, Murcia SPAIN

Corresponding Author Secondary Information:

Corresponding Author's Institution:

Santa Lucia University Hospital

Corresponding Author's Secondary Institution:

First Author:

Silvia Montoro-Garcia, Ph.D

First Author Secondary Information:

Order of Authors:

Silvia Montoro-Garcia, Ph.D

Begoña Alburquerque-González, BSc

Ángel Bernabé-García, Ph.D

Manuel Bernabé-García, Ph.D

Priscila Campioni Rodrigues, Ph.D

Helena den Haan, Ph.D

Irene Luque, Ph.D

Francisco José Nicolas, Ph.D

Horacio Pérez-Sánchez, Ph.D

María Luisa Cayuela, Ph.D

Tuula Salo, Ph.D

Pablo Conesa-Zamora, Ph.D, Pharm.D

Order of Authors Secondary Information:

Abstract:

Tumor invasion and metastasis involve processes in which actin cytoskeleton rearrangement induced by Fascin1 plays a crucial role. Indeed, Fascin1 has been found overexpressed in tumors with worse prognosis. Migrastatin and its analogues target Fascin1 and inhibit its activity. However, there is need for novel and smaller Fascin 1 inhibitors. The aim of this study was to assess the effect of compound G2 in colorectal cancer cell lines and compare it to migrastatin in in vitro and in vivo assays. Molecular modeling, actin bundling, cell viability, inmunofluorescence, migration and invasion assays were carried out in order to test anti-migratory and antiinvasive properties of compound G2. In addition, the in vivo effect of compound G2 was evaluated in a zebrafish model of invasion. HCT-116 cells exhibited the highest Fascin1 expression from eight tested colorectal cancer cell lines. Compound G2 showed important inhibitory effects on actin bundling, filopodia formation, migration and invasion in different cell lines. Moreover, compound G2 treatment resulted in significant reduction of invasion of DLD-1 overexpressing Fascin1 and HCT-116 in zebrafish larvae xenografts; this effect being less evident in Fascin1 known-down HCT116 cells. This study proves, for the first time, the in vitro and in vivo antitumoral activity of compound G2 on colorectal cancer cells and guides to design improved compound G2-based Fascin1 inhibitors. 
General remarks:

Careful proofreading is necessary.

REPLY: The manuscript has been now reviewed carefully by a native English speaker. It is required to use the same cell lines for each assay: HCT-116 control + HCT-116

fascin siRNA and DLD1 control + fascin overexpression.

REPLY: We understand the referee's comment and we have now performed the corresponding experiments accordingly. All of them are now included in the new version of the manuscript (main text and supplemental material).

We are now describing to the referee the main issues about the above assays with transfected cells.

Figure 2. This is not new but has been already shown by Huang et al., 2015. The evaluation method to analyze the effect of the compounds on fascin is not clear and it is not possible to read the graph labeling.

REPLY: Huang et al. (2018) performed elegant experiments to identify small molecules that specifically block filopodia formation, cell migration and invasion. Compound G2 was among these molecules. They appropriately used fluorescence microscopy $(63 x$ magnification) together with isothermal titration calorimetry to test the biochemical mechanism by which $\mathrm{G} 2$ inhibits fascin1. Nonetheless, transmission electronic microscopy (TEM) $(135,000 x$ magnification), as we presented here, was not used in the referred paper.

Moreover, we have now modified the graph labelling in Figure 2.

Huang, J., Dey, R., Wang, Y., Jakoncic, J., Kurinov, I., \& Huang, X. Y. (2018). Structural Insights into the Induced-fit Inhibition of Fascin by a Small-Molecule Inhibitor. J Mol Biol, 430(9), 1324-1335. doi:10.1016/j.jmb.2018.03.009

Figure 3: Only HCT-116 cells were analyzed the other cell lines are missing, see "general remarks"

REPLY: Not all cell lines are suitable to assess filopodia formation. In order to provide an extra cell line to demonstrate the effect of compound G2, HaCaT human cells were used. HaCaT are spontaneously immortalized human keratinocyte cells expressing high levels of fascin1 (https://www.proteinatlas.org/ENSG00000075618-FSCN1/cell). As it is shown in the following figure (for reviewer only), lamellipodia formation in the presence of compound G2 was clearly abolished in HaCat cells, confirming once more the implication of the drug in the lamellipodia formation.

Figure for review only: Representative images of immunofluorescence assaysof HaCat cells for fascin1 (green), nucleus (blue) and actin (red) are shown. A) Control condition (DMSO); B) $10 \mathrm{ng} / \mathrm{mL}$ Epidermal Growth Factor (EGF, migration stimulator); C) $50 \mu \mathrm{M}$ PD98059 (Mek inhibitor); D) $100 \mu \mathrm{M}$ migrastatin; E) $10 \mu \mathrm{M}$ compound G2. Cells were fixed with bouin and co-stained with anti-Fascin1 antibody (1/250) and Hoechst-33258. For actin staining, cells were fixed with methanol and stained with anti-actin antibody (1/1000) and Hoechst-33258. Images were captured with a LSM 510 META confocal fluorescence microscope with $63 X$ oil objective.

RESULTS (PAGE 14): "Similar results were observed with an extra cell line expressing Fascin1 (HaCat) as DLD1 morphology was not suitable for assessing lamellipodium formation (data not shown)".

Additionally, we now include the immunofluorescence assay with genetically knockeddown HCT-116 for fascin1 (Supplementary material S5). The resulting experiment shows that lamellipodia formation is clearly compromised in fascin 1 silenced cells and therefore, the effect of $\mathrm{G} 2$ cannot be exerted in an additive fashion. Nonetheless, it is important to notice that $100 \%$ transfection efficiency was not achieved and thus, some cells are stained in green and still display lamellipodia.

When pharmacological inhibition (compound G2) is added to knocked-down cells, lamellipodia are also abolished. The following figure includes the effect of compound G2 on silenced cells. Nonetheless, in the supplementary material we have only added columns A-D because the lamellipodia formation in silenced cells was already abolished without the drug.

Figure for review only: Representative images of immunofluorescence assays for fascin1 (green), nucleus (blue) and actin (red) are shown. A) Control condition (DMSO) with siRNA-A transfected HCT-116 cells; B) Control condition (DMSO) with fascin1 silenced HCT-116 cells; C) Fascin 1 silenced HCT-116 cells with $10 \mathrm{ng} / \mathrm{mL}$ Epidermal Growth Factor (EGF, migration stimulator), D) Fascin1 silenced HCT-116 cells with 50 $\mu \mathrm{M}$ PD98059 (Mek inhibitor); E) Fascin1 silenced HCT-116 cells with $20 \mu \mathrm{M}$ compound G2. Colorectal carcinoma HCT-116 cells were genetically knocked-down for Fascin1 
with short interfering siRNA, control HCT-116 was transfected with siRNA-A (MOCK). Cells were fixed with bouin and co-stained with anti-Fascin1 antibody (1/250) and Hoechst-33258. For actin staining, cells were fixed with methanol and stained with antiactin antibody (1/1000). Images were captured with a LSM 510 META confocal fluorescence microscope with $63 \mathrm{X}$ oil objective.

Figure 4: The siRNA and overexpressing approaches are missing. Figure 5: Only HCT116 cells were analyzed the other cell lines are missing, see "general remarks" REPLY: As suggested by the referee, a new supplemental figure (S9) has been included presenting new migration and invasion assays using HCT-116 control + HCT116 fascin1 siRNA and DLD1 control + fascin1 overexpression. In this way, genetic and pharmacological inhibitions could be analyzed in vitro.

Figure for review only: Scratch of transfected DLD-1 cells with pGFP-Fsc1. Fascin1 silenced HCT-116 cells lead to a slightly decrease in migration and invasion compared to MOCK HCT-116 cells, being more significant in the presence of compound G2. Similarly, DLD1 overexpressing Fascin1 migrate and invade significantly more than MOCK DLD-1 cells whilst treated DLD1 cells display reduced capacities.

Supplementary material S9. Inhibition of the migration and invasive capacities exerted by compound $\mathrm{G} 2$ on transfected cells Colorectal carcinoma HCT-116 cells were genetically knocked-down for Fascin1 with short interfering siRNA, MOCK control HCT116 was transfected with siRNA-A: A) Percentage of migration. B) Percentage of invasion. Colorectal carcinoma DLD-1 cells were genetically overexpressed for Fascin1 with Fascin1-GFP vector, MOCK control DLD-1 was transfected with pGFP N3 control vector: C) Percentage of migration. D) Percentage of invasion Data are representative of two similar experiments Error bars, mean \pm SD of duplicates. * $p<0.05,{ }^{* *} p<0.01$ compared to MOCK condition; n.s: non-significant.

Figure 6: "Good correlation was observed between Fascin1 expression and cell invasion". This is not true HCT-15 cells exhibit higher fascin mRNA levels than DLD1 cells and there is not difference between these cell lines.

REPLY: The reviewer's comment is pertinent. This statement can be applied for most but not all cell lines assayed. Therefore, the sentence has been reworded as follows: RESULTS (PAGE 18): "Good correlation was observed between Fascin1 expression and cell invasion for all cell lines assayed except HCT-15 whose invasion was lower than expected possibly due to other fascin 1 independent factors affecting invasion (Figure 6B). Therefore, and because of good correlation, HCT-116 was selected for being the cell line with the highest and DLD-1 for its lower Fascin1 expression and cell invasion."

Figure 7: These experiments have been properly done. However, there are still some concerns. Figure 7C. There is no significant (n.s.) difference between siRNA control cells treated with 5 or $10 \mu \mathrm{M} \mathrm{G} 2$. Why is the dose-dependency missing in Fascinexpressing cells? The same is true for Figure $7 \mathrm{~F}$, in fascin expressing cells one would expect a dose-dependency.

REPLY: We understand the reviewer's concerns about the dose-dependency relationship in transfected cells. Migration and invasion are complex processes involving other proteins and factors apart from fascin 1 . The invasion capacity cannot be only associated with fascin1 levels.

The expression of fascin1 in silenced HCT-116 cells was low and with such low levels, it was difficult to find a clear dose-dependent relationship of an inhibitory compound (although a trend was shown). Nevertheless, the demonstration that the effect of G2 is fascin1 dependent comes from the comparison between siRNA fascin1 HCT-116 and Fascin1 transfected DLD1 (Fig 7C and 7F) where the inhibitory effect of G2 on invasion is maximum when fascin 1 is overexpressed and minimum when it is silenced.

Accordingly, this inhibitory effect is intermediate when fascin 1 expression is not altered by transfection. It is clear that close concentrations (i.e. 5 and 10uM) can result in nonsignificant relationship although the tendency is visible by comparing the bar heights. Although a significant effect compared to MOCK conditions was found, the absence of this tendency in Fascin1 transfected DLD1 (Fig. 7F) could be explained by the fact that the $\mathrm{G} 2$ inhibitory activity is in its maximum (regardless 5 or $10 \mathrm{uM} \mathrm{G2}$ ) because under this circumstance, invasion is more dependent on Fascin1 (which is exogenously overexpressed) compared to the non-transfected condition that is closer to the in vivo condition. Nonetheless, there is a common anti-invasive phenotype when either pharmacological or genetic inhibition are performed in HCT-116 cells. Huang et al 


\begin{tabular}{|c|c|c|}
\hline & \multicolumn{2}{|c|}{ 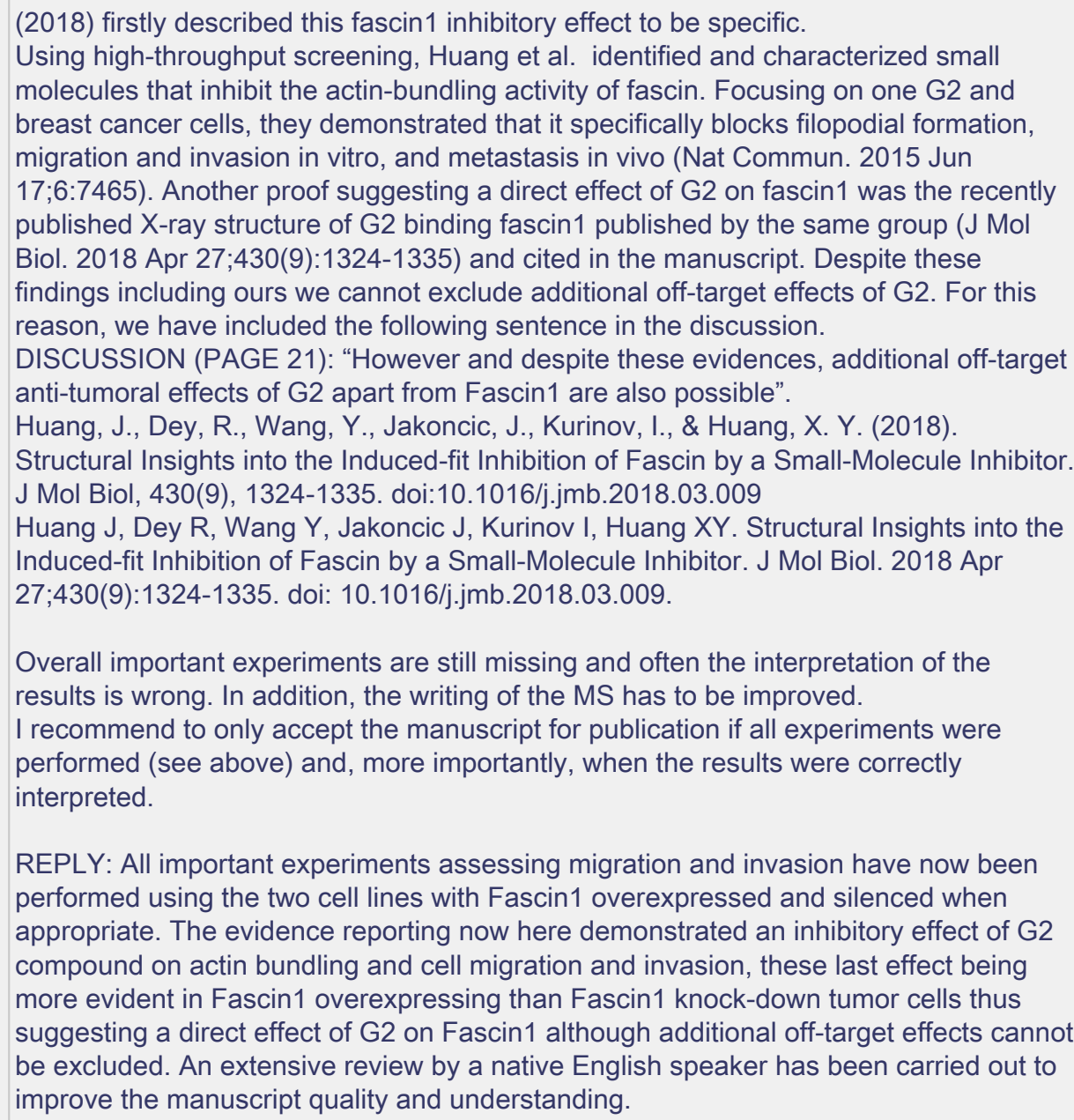 } \\
\hline \multirow[t]{5}{*}{ Funding Information: } & $\begin{array}{l}\text { Instituto de Salud Carlos III } \\
\text { (PI12/1232) }\end{array}$ & Dr. Pablo Conesa-Zamora \\
\hline & $\begin{array}{l}\text { Instituto de Salud Carlos III } \\
\text { (PI15/00626) }\end{array}$ & Dr. Pablo Conesa-Zamora \\
\hline & $\begin{array}{l}\text { Ministerio de Economía, Industria y } \\
\text { Competitividad, Gobierno de España } \\
\text { (CTQ2017-87974-R) }\end{array}$ & Dr. Horacio Pérez-Sánchez \\
\hline & $\begin{array}{l}\text { Fundación Séneca } \\
(18946 / J L I / 13)\end{array}$ & Dr. Horacio Pérez-Sánchez \\
\hline & $\begin{array}{l}\text { H2020 Marie Skłodowska-Curie Actions } \\
\text { (FP7/2007-2013) }\end{array}$ & Dr Silvia Montoro-Garcia \\
\hline
\end{tabular}




\section{General remarks:}

Careful proofreading is necessary.

REPLY: The manuscript has been now reviewed carefully by a native English speaker.

It is required to use the same cell lines for each assay: HCT-116 control + HCT-116 fascin siRNA and DLD1 control + fascin overexpression.

REPLY: We understand the referee's comment and we have now performed the corresponding experiments accordingly. All of them are now included in the new version of the manuscript (main text and supplemental material).

We are now describing to the referee the main issues about the above assays with transfected cells.

Figure 2. This is not new but has been already shown by Huang et al., 2015. The evaluation method to analyze the effect of the compounds on fascin is not clear and it is not possible to read the graph labeling.

REPLY: Huang et al. (2018) performed elegant experiments to identify small molecules that specifically block filopodia formation, cell migration and invasion. Compound G2 was among these molecules. They appropriately used fluorescence microscopy (63x magnification) together with isothermal titration calorimetry to test the biochemical mechanism by which $\mathrm{G} 2$ inhibits fascin1. Nonetheless, transmission electronic microscopy (TEM) $(\mathbf{1 3 5 , 0 0 0 x}$ magnification), as we presented here, was not used in the referred paper.

Moreover, we have now modified the graph labelling in Figure 2.

Huang, J., Dey, R., Wang, Y., Jakoncic, J., Kurinov, I., \& Huang, X. Y. (2018). Structural Insights into the Induced-fit Inhibition of Fascin by a Small-Molecule Inhibitor. J Mol Biol, 430(9), 1324-1335. doi:10.1016/j.jmb.2018.03.009

Figure 3: Only HCT-116 cells were analyzed the other cell lines are missing, see "general remarks"

REPLY: Not all cell lines are suitable to assess filopodia formation. In order to provide an extra cell line to demonstrate the effect of compound G2, HaCaT human cells were used. $\mathrm{HaCaT}$ are spontaneously immortalized human keratinocyte cells expressing high levels of fascin1 (https://www.proteinatlas.org/ENSG00000075618-FSCN1/cell). As it is shown in the following figure (for reviewer only), lamellipodia formation in the presence of compound G2 was clearly abolished in HaCat cells, confirming once more the implication of the drug in the lamellipodia formation. 

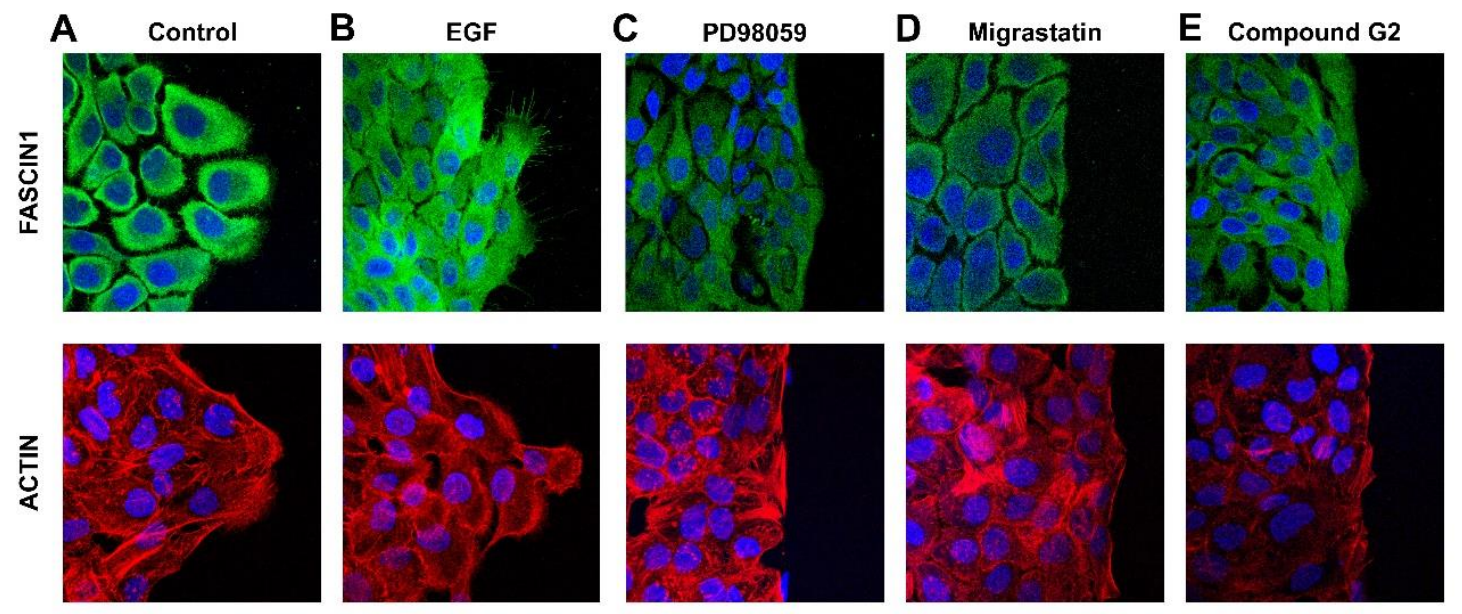

Figure for review only: Representative images of immunofluorescence assaysof HaCat cells for fascin1 (green), nucleus (blue) and actin (red) are shown. A) Control condition (DMSO); B) 10 $\mathrm{ng} / \mathrm{mL}$ Epidermal Growth Factor (EGF, migration stimulator); C) $50 \mu \mathrm{M}$ PD98059 (Mek inhibitor); D) $100 \mu \mathrm{M}$ migrastatin; E) $10 \mu \mathrm{M}$ compound G2. Cells were fixed with bouin and costained with anti-Fascin1 antibody (1/250) and Hoechst-33258. For actin staining, cells were fixed with methanol and stained with anti-actin antibody (1/1000) and Hoechst-33258. Images were captured with a LSM 510 META confocal fluorescence microscope with 63X oil objective.

RESULTS (PAGE 14): "Similar results were observed with an extra cell line expressing Fascin1 (HaCat) as DLD1 morphology was not suitable for assessing lamellipodium formation (data not shown)".

Additionally, we now include the immunofluorescence assay with genetically knocked-down HCT-116 for fascin1 (Supplementary material S5). The resulting experiment shows that lamellipodia formation is clearly compromised in fascin1 silenced cells and therefore, the effect of $\mathbf{G} 2$ cannot be exerted in an additive fashion. Nonetheless, it is important to notice that $100 \%$ transfection efficiency was not achieved and thus, some cells are stained in green and still display lamellipodia.

When pharmacological inhibition (compound G2) is added to knocked-down cells, lamellipodia are also abolished. The following figure includes the effect of compound $G 2$ on silenced cells. Nonetheless, in the supplementary material we have only added columns A-D because the lamellipodia formation in silenced cells was already abolished without the drug. 
A
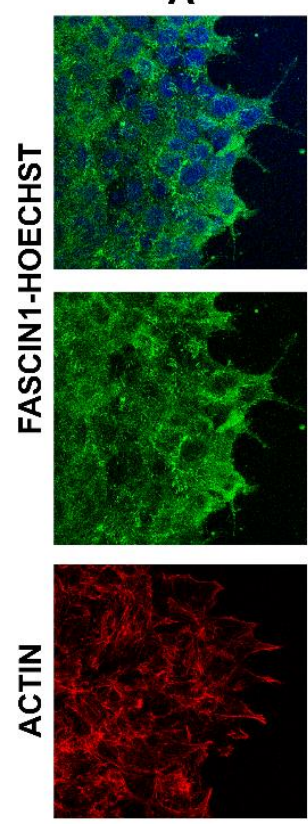

B
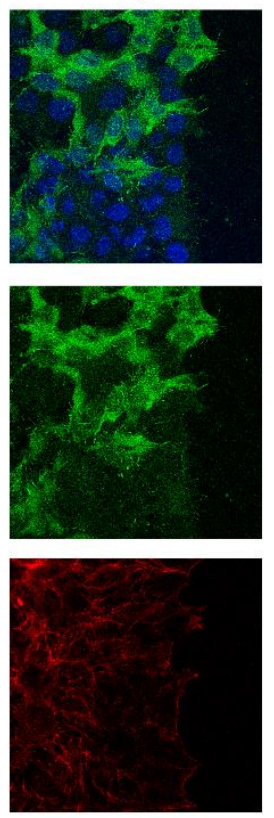

C
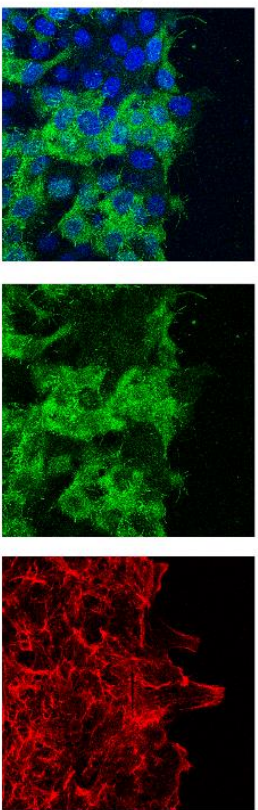

D
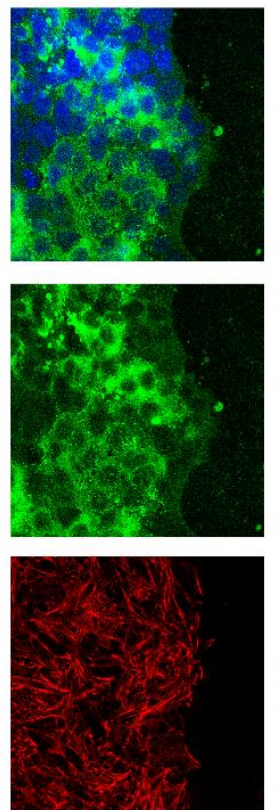

$\mathbf{E}$
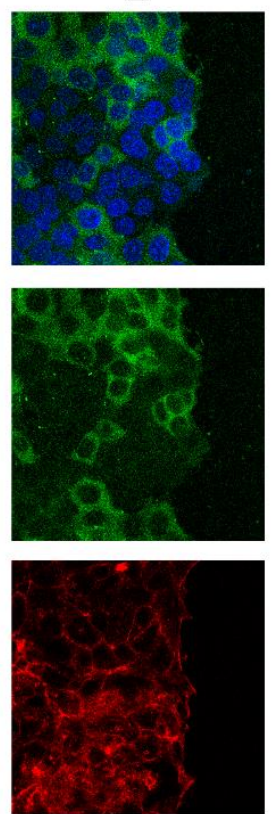

Figure for review only: Representative images of immunofluorescence assays for fascin1 (green), nucleus (blue) and actin (red) are shown. A) Control condition (DMSO) with siRNA-A transfected HCT-116 cells; B) Control condition (DMSO) with fascin1 silenced HCT-116 cells; C) Fascin1 silenced HCT-116 cells with $10 \mathrm{ng} / \mathrm{mL}$ Epidermal Growth Factor (EGF, migration stimulator), D) Fascin1 silenced HCT-116 cells with $50 \mu$ M PD98059 (Mek inhibitor); E) Fascin1 silenced HCT-116 cells with $20 \mu \mathrm{M}$ compound G2. Colorectal carcinoma HCT-116 cells were genetically knocked-down for Fascin1 with short interfering siRNA, control HCT-116 was transfected with siRNA-A (MOCK). Cells were fixed with bouin and co-stained with anti-Fascin1 antibody (1/250) and Hoechst-33258. For actin staining, cells were fixed with methanol and stained with anti-actin antibody (1/1000). Images were captured with a LSM 510 META confocal fluorescence microscope with 63X oil objective.

Figure 4: The siRNA and overexpressing approaches are missing. Figure 5: Only HCT-116 cells were analyzed the other cell lines are missing, see "general remarks"

REPLY: As suggested by the referee, a new supplemental figure (S9) has been included presenting new migration and invasion assays using HCT-116 control + HCT-116 fascin1 siRNA and DLD1 control + fascin1 overexpression. In this way, genetic and pharmacological inhibitions could be analyzed in vitro. 


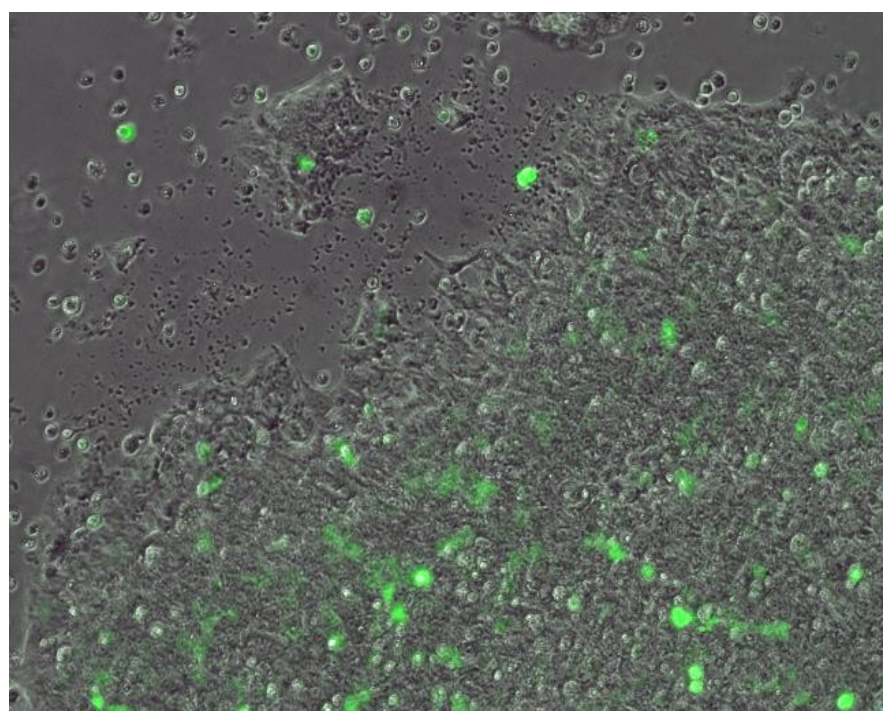

Figure for review only: Scratch of transfected DLD-1 cells with pGFP-Fsc1.

Fascin1 silenced HCT-116 cells lead to a slightly decrease in migration and invasion compared to MOCK HCT-116 cells, being more significant in the presence of compound G2. Similarly, DLD1 overexpressing Fascin1 migrate and invade significantly more than MOCK DLD-1 cells whilst treated DLD1 cells display reduced capacities.
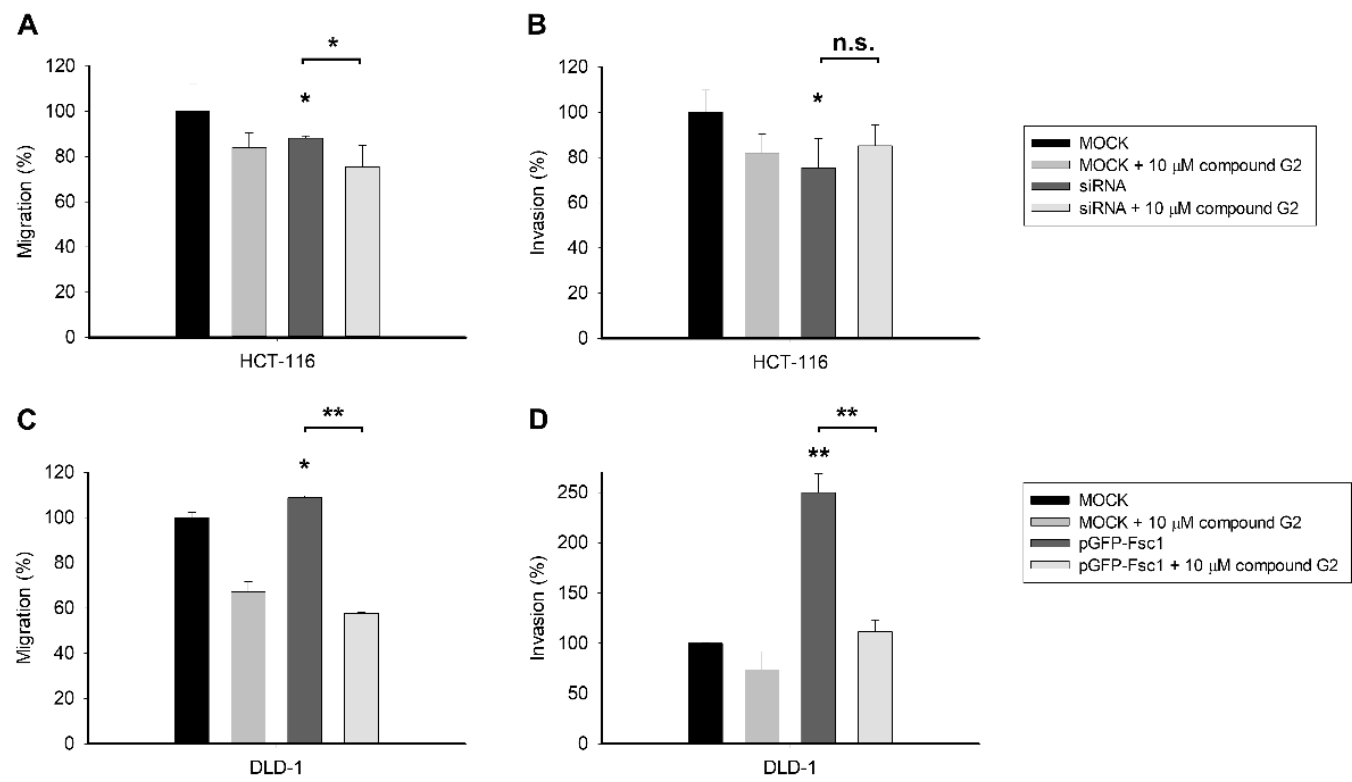

Supplementary material S9. Inhibition of the migration and invasive capacities exerted by compound G2 on transfected cells Colorectal carcinoma HCT-116 cells were genetically knocked-down for Fascin1 with short interfering siRNA, MOCK control HCT-116 was transfected with siRNA-A: A) Percentage of migration. B) Percentage of invasion. Colorectal carcinoma DLD-1 cells were genetically overexpressed for Fascin1 with Fascin1-GFP vector, MOCK control DLD-1 was transfected with pGFP N3 control vector: C) Percentage of migration. D) Percentage of invasion Data are representative of two similar experiments Error bars, mean \pm SD of duplicates. ${ }^{*} p<0.05,{ }^{* *} p<0.01$ compared to MOCK condition; n.s: non-significant. 
Figure 6: "Good correlation was observed between Fascin1 expression and cell invasion". This is not true HCT-15 cells exhibit higher fascin mRNA levels than DLD1 cells and there is not difference between these cell lines.

REPLY: The reviewer's comment is pertinent. This statement can be applied for most but not all cell lines assayed. Therefore, the sentence has been reworded as follows:

RESULTS (PAGE 18): "Good correlation was observed between Fascin1 expression and cell invasion for all cell lines assayed except HCT-15 whose invasion was lower than expected possibly due to other fascin1 independent factors affecting invasion (Figure 6B). Therefore, and because of good correlation, HCT-116 was selected for being the cell line with the highest and DLD-1 for its lower Fascin1 expression and cell invasion."

Figure 7: These experiments have been properly done. However, there are still some concerns. Figure 7C. There is no significant (n.s.) difference between siRNA control cells treated with 5 or $10 \mu \mathrm{M} \mathrm{G2}$. Why is the dose-dependency missing in Fascin-expressing cells? The same is true for Figure 7F, in fascin expressing cells one would expect a dose-dependency.

REPLY: We understand the reviewer's concerns about the dose-dependency relationship in transfected cells. Migration and invasion are complex processes involving other proteins and factors apart from fascin1. The invasion capacity cannot be only associated with fascin1 levels.

The expression of fascin1 in silenced HCT-116 cells was low and with such low levels, it was difficult to find a clear dose-dependent relationship of an inhibitory compound (although a trend was shown). Nevertheless, the demonstration that the effect of G2 is fascin 1 dependent comes from the comparison between siRNA fascin1 HCT-116 and Fascin1 transfected DLD1 (Fig $7 C$ and $7 F$ ) where the inhibitory effect of $G 2$ on invasion is maximum when fascin 1 is overexpressed and minimum when it is silenced. Accordingly, this inhibitory effect is intermediate when fascin 1 expression is not altered by transfection. It is clear that close concentrations (i.e. 5 and $10 \mathrm{uM}$ ) can result in non-significant relationship although the tendency is visible by comparing the bar heights. Although a significant effect compared to MOCK conditions was found, the absence of this tendency in Fascin1 transfected DLD1 (Fig. 7F) could be explained by the fact that the G2 inhibitory activity is in its maximum (regardless 5 or 10 uM G2) because under this circumstance, invasion is more dependent on Fascin1 (which is exogenously overexpressed) compared to the non-transfected condition that is closer to the in vivo condition. Nonetheless, there is a common anti-invasive phenotype when either pharmacological or genetic inhibition are performed in HCT-116 cells. Huang et al (2018) firstly described this fascin1 inhibitory effect to be specific.

Using high-throughput screening, Huang et al. identified and characterized small molecules that inhibit the actin-bundling activity of fascin. Focusing on one G2 and breast cancer cells, they demonstrated that it specifically blocks filopodial formation, migration and invasion in vitro, and metastasis in vivo (Nat Commun. 2015 Jun 17;6:7465). Another proof suggesting a direct effect of $\mathrm{G} 2$ on fascin1 was the recently published X-ray structure of $\mathrm{G} 2$ binding fascin 1 published by the same group (J Mol Biol. 2018 Apr 27;430(9):1324-1335) and cited in the manuscript. Despite these findings including ours we cannot exclude additional off-target effects of G2. For this reason, we have included the following sentence in the discussion.

DISCUSSION (PAGE 21): "However and despite these evidences, additional off-target antitumoral effects of $\mathrm{G} 2$ apart from Fascin1 are also possible". 
Huang, J., Dey, R., Wang, Y., Jakoncic, J., Kurinov, I., \& Huang, X. Y. (2018). Structural Insights into the Induced-fit Inhibition of Fascin by a Small-Molecule Inhibitor. J Mol Biol, 430(9), 1324-1335. doi:10.1016/j.jmb.2018.03.009

Huang J, Dey R, Wang Y, Jakoncic J, Kurinov I, Huang XY. Structural Insights into the Induced-fit Inhibition of Fascin by a Small-Molecule Inhibitor. J Mol Biol. 2018 Apr 27;430(9):1324-1335. doi: 10.1016/j.jmb.2018.03.009.

Overall important experiments are still missing and often the interpretation of the results is wrong. In addition, the writing of the MS has to be improved.

I recommend to only accept the manuscript for publication if all experiments were performed (see above) and, more importantly, when the results were correctly interpreted.

REPLY: All important experiments assessing migration and invasion have now been performed using the two cell lines with Fascin1 overexpressed and silenced when appropriate. The evidence reporting now here demonstrated an inhibitory effect of G2 compound on actin bundling and cell migration and invasion, these last effect being more evident in Fascin1 overexpressing than Fascin1 knock-down tumor cells thus suggesting a direct effect of $G 2$ on Fascin1 although additional off-target effects cannot be excluded. An extensive review by a native English speaker has been carried out to improve the manuscript quality and understanding. 
Dear Editor,

Please find enclosed the revised version of our work: "Novel anti-migratory and antiinvasive properties of a Fascin1 inhibitor on colorectal cancer cells"

In the current version of the manuscript, we have given point-by-point response to all reviewer's questions for which we have performed new assays requested by the reviewer in his second revision. These new experiments includes, when appropriate, silencing and overexpression of fascin 1 in HCT-116 and DLD1 cell lines, respectively in order to assess the effect of G2 compound on migration and invasion of tumor cells both in vitro and in vivo. Besides, we show that G2 disrupts the fascin-actin filament interactions (transmission electron microscopy) and cellular lamelipodia formation (confocal microscopy) in colorectal cancer cells. Text changes from the first revision are highlighted in yellow and from the second revision in light blue.

During more than eight years our group have been characterizing the molecular features of serrated pathway of the colorectum and found out that Fascin 1 was an interesting molecular target. This study reports for the first time that a Fascin 1 inhibitor has anti-migratory and anti-invasive properties in colorectal cancer cells even at lower concentrations than migrastatin, one the most characterized Fascin1 inhibitor. Using this strategy, we have here confirmed a novel class of compounds that can be a foundation for treating cancer characterized by fascin 1 overexpression such as Serrated Adenocarcinoma of the colorectum. The study provides significant evidence that G2 compound is an interesting candidate for further investigation/chemical modifications to develop new Fascin1-specific therapies for colorectal cancer and constitutes the first rationale for a tailored therapy in serrated adenocarcinoma.

Hospital General Universitario

Santa Lucía

C/ Mezquita s/n, Paraje los Arcos 
All authors have contributed to and agreed on the content of the manuscript, and the respective roles of each author. The manuscript is original and has not been published previously, in any language, in whole or in part, and is not currently under consideration elsewhere. The study was approved by the Hospital Ethics Committee and was carried out in accordance with the ethical standards laid down in the 1964 Declaration of Helsinki and its later amendments.

Your comments and suggestions will be greatly appreciated.

Yours faithfully.

Pablo Conesa Zamora, PharmD. 


\section{Key messages}

-Fascin is crucial for tumor invasion and metastasis and is overexpressed in bad prognostic tumors

-Several adverse tumors overexpress Fascin1 and lack targeted therapy

-Anti-fascin G2 is for the first time evaluated in colorectal carcinoma and compared with migrastatin

-Filopodia formation, migration activity and invasion in vitro and in vivo assays were performed

-G2 blocks actin structures, migration and invasion of colorectal cancer cells as fascin dependent 


\section{Novel anti-invasive properties of a Fascin1 inhibitor on colorectal cancer cells}

Silvia Montoro-García ${ }^{a *}$, Begoña Alburquerque-González ${ }^{\mathrm{b} *}$, Ángel Bernabé-García ${ }^{\mathrm{c}}$, Manuel Bernabé-García ${ }^{\mathrm{d}}$, Priscila Campioni Rodrigues ${ }^{\mathrm{e}}$, Helena den-Haan ${ }^{\mathrm{f}}$, Irene Luque $^{\mathrm{g}}$, Francisco José Nicolas ${ }^{\mathrm{c}}$, Horacio Pérez-Sánchez ${ }^{\mathrm{h}}$, María Luisa Cayuela ${ }^{\mathrm{d}}$, Tuula Salo $^{\mathrm{e}, \mathrm{i}}$, Pablo Conesa-Zamora ${ }^{\mathrm{b}, \mathrm{j}}$

*Equally contributed

a. Cátedra de Riesgo Cardiovascular, Facultad de Ciencias de la Salud, UCAM Universidad Católica San Antonio de Murcia, Campus de los Jerónimos, s/n, Guadalupe 30107 Murcia, Spain.

b. Pathology and Histology Department Facultad de Ciencias de la Salud, UCAM Universidad Católica San Antonio de Murcia, Campus de los Jerónimos, s/n, Guadalupe 30107 Murcia, Spain.

c. Laboratorio de Regeneración, Oncología Molecular y TGF-ß3. Biomedical Research Institute of Murcia (IMIB-Arrixaca), Carretera Madrid-Cartagena. El Palmar, Murcia, Spain.

d. Telomerase, Cancer and Aging Group, University Clinical Hospital "Virgen de la Arrixaca”, Biomedical Research Institute of Murcia (IMIB-Arrixaca) Murcia, Spain.

e. Cancer and Translational Medicine Research Unit, Faculty of Medicine, University of Oulu, Aapistie 5A, FI-90220, Oulu, Finland and Medical Research Center Oulu, Oulu University Hospital, University of Oulu, Oulu, Finland. Priscila.CampioniRodrigues@oulu.fi 
f. Eurofins Villapharma Research, Parque Tecnológico de Fuente Álamo. Ctra. El Estrecho-Lobosillo, Km 2,5. Av. Azul E, 30320, Murcia, Spain.

g. Department of Physical Chemistry and Institute of Biotechnology, University of Granada, Campus Fuentenueva s/n 18071 Granada, Spain.

h. Structural Bioinformatics and High Performance Computing (BIO-HPC) Research Group, Universidad Católica de Murcia (UCAM), Guadalupe, Spain.

i. Institute of Oral and Maxillofacial Disease, University of Helsinki, and HUSLAB, Department of Pathology, Helsinki University Hospital, Helsinki, Finland.

j. Clinical Analysis Department, Group of Molecular Pathology and Pharmacogenetics, Biomedical Research Institute from Murcia (IMIB), Hospital Universitario Santa Lucía, c/Mezquita sn, 30202 Cartagena, Spain.

Corresponding authors:

Silvia Montoro-Garcia. Campus de los Jerónimos, s/n, Guadalupe 30107 Murcia, Spain. Tel: +34 968278655. Email: smontoro@ucam.edu. Orcid: orcid.org/0000-0002-1948$\underline{1022}$

Pablo Conesa-Zamora. c/Mezquita sn, 30202 Cartagena, Spain. Tel +34 968128602 Ext 951615. Email: pablo.conesa@ carm.es. Orcid: orcid.org/0000-0003-0190-3044

\section{Conflict of interest statement}

The authors confirm that there are no conflicts of interest. 


\section{Acknowledgments}

This project received grants from Instituto de Salud Carlos III (Spanish Ministry of Health) and FEDER funds (ref: PI12/1232 and PI15/00626), Spanish Ministry of Economy and Competitiveness MINECO (CTQ2017-87974-R), and by the Fundación Séneca del Centro de Coordinación de la Investigación de la Región de Murcia under Projects 18946/JLI/13 and 20646/JLI/18. BAG belongs to the "Programa de Doctorado en Ciencias de la Salud, Universidad Católica de Murcia (UCAM)" and holds a grant of the UCAM. PCR was supported by Finnish Cultural Foundation Grant (2017-2018). This research was partially supported by the e-infrastructure program of the Research Council of Norway, and the supercomputer center of UiT - the Arctic University of Norway, and by the supercomputing infrastructure of Poznan Supercomputing Center. 


\begin{abstract}
Tumor invasion and metastasis involve processes in which actin cytoskeleton rearrangement induced by Fascin1 plays a crucial role. Indeed, Fascin1 has been found overexpressed in tumors with worse prognosis. Migrastatin and its analogues target Fascin 1 and inhibit its activity. However, there is need for novel and smaller Fascin1 inhibitors. The aim of this study was to assess the effect of compound G2 in colorectal cancer cell lines and compare it to migrastatin in in vitro and in vivo assays. Molecular modeling, actin bundling, cell viability, inmunofluorescence, migration and invasion assays were carried out in order to test anti-migratory and anti-invasive properties of compound G2. In addition, the in vivo effect of compound G2 was evaluated in a zebrafish model of invasion. HCT-116 cells exhibited the highest Fascin1 expression from eight tested colorectal cancer cell lines. Compound G2 showed important inhibitory effects on actin bundling, filopodia formation, migration and invasion in different cell lines. Moreover, compound G2 treatment resulted in significant reduction of invasion of DLD-1 overexpressing Fascin1 and HCT-116 in zebrafish larvae xenografts; this effect being less evident in Fascin1 known-down HCT-116 cells. This study proves, for the first time, the in vitro and in vivo antitumoral activity of compound G2 on colorectal cancer cells and guides to design improved compound G2-based Fascin1 inhibitors.
\end{abstract}

Keywords: Fascin1; Migrastatin; Invasion; Migration; Zebrafish xenograft; Colorectal Cancer. 


\section{Introduction}

Tumor metastasis remains the leading cause of cancer mortality (Chen, Yang, Jakoncic, Zhang, \& Huang, 2010). Acquisition of invading capacity is a prerequisite for carcinoma cells to get access to vessels and thus spread throughout the body. This process involves actin cytoskeleton rearrangement allowing the tumor cells to develop cellular protrusions, such as filopodia and lamellipodium, which contribute to cancer cell migration, invasion, and metastasis (Machesky \& Li, 2010). Fascin1 (FSCN1: ENSG00000075618) is a key protein in membrane protrusion, as it possesses actinbinding and actin-bundling activity by cross-linking filamentous actin into tightly packed parallel bundles. Fascin1 expression is often elevated in malignant tumors while its expression is low or absent in most normal adult epithelia (Hashimoto, Kim, \& Adams, 2011). Fascin1 has emerged as an important biomarker and therapeutic target due to its overexpression in several carcinomas and its association with mortality and metastasis (Hashimoto et al., 2011; Hashimoto, Skacel, \& Adams, 2005). In fact, several studies including a meta-analysis have demonstrated that Fascin 1 expression is associated with increased lymph node- and distant-metastasis, disease progression and mortality in both colorectal and breast cancer (Omran \& Al Sheeha, 2015; Tan, Lewis, Adams, \& Martin, 2013).

In a recent study, our group identified Fascin1 as overexpressed in serrated adenocarcinoma (SAC) (Conesa-Zamora et al., 2013), a histological subtype of colorectal carcinoma. SAC, in contrast to conventional colorectal carcinoma (GarcíaSolano, Conesa-Zamora, Trujillo-Santos, et al., 2012), has worse prognosis (GarcíaSolano et al., 2010) and it is characterized by a more active invasive front evidenced by higher occurrence of tumor budding, cytoplasmic pseudofragments, infiltrative tumor growth pattern (García-Solano, Conesa-Zamora, Trujillo-Santos, Mäkinen, \& Pérez- 
Guillermo, 2011) and E-cadherin loss. Moreover, SACs show a higher frequency of KRAS and BRAF mutations than conventional carcinoma which make most of them resistant to anti-EGFR therapy (García-Solano, Conesa-Zamora, Carbonell, et al., 2012; Stefanius et al., 2011).

Given the causative role of Fascin1 in the invading phenotype of tumor cells together with the association of its overexpression to worse survival of a wide variety of cancer types (Cao et al., 2014; Jones et al., 2015; Li et al., 2014; Rodrigues et al., 2017; Stewart \& Crook, 2015; Zhao et al., 2015) it would be desirable to find efficient Fascin1-activity blockers. In this line, migrastatin and its macroketone analogues are considered typical Fascin1 inhibitors (Chen et al., 2010). Unfortunately, they are difficult to synthesize due to their complex structure (Gaul et al., 2004).

In this study, we performed a search of patented potential Fascin 1 inhibitors, such as those derived from indazol-furan-carboxamides (Han et al., 2016). Among them, we found the leading compound G2 and showed an inhibitory effect on Fascin 1 activity by using several in vitro and in vivo assays on well-characterized colorectal cell lines.

\section{Material and Methods}

\section{Compounds and molecular modeling}

Compound G2 (N-(1-(4-(trifluoromethyl) benzyl)-1H-indazol-3-yl) furan-2carboxamide; C20H15F3N3O2; PM 386.13) is covered by the patents WO 2014/031732 A2 and WO 2015/127125A1 and was synthesized as previously described (Han et al., 2016). Migrastatin was synthesized by AnalytiCon Discovery (NP-006108) and provided by MolPort (Riga, Latvia). The geometry of compound G2 was built with 
Autodock Tools (Morris et al., 2009), where partial charges were assigned using Gasteiger model (Gasteiger \& Marsili, 1980). The structural model for Fascin1 was extracted from the crystal structure of protein data bank (PDB) with code 6B0T (Huang et al., 2018) and converted to PDBQT format using default parameters. Molecular docking calculations based on the Blind Docking (BD) technique (Sánchez-Linares, Pérez-Sánchez, Cecilia, \& García, 2012) were carried out using Blind Docking server (BDS, available at http://bio-hpc.ucam.edu/achilles) with Autodock 4 (Morris et al., 2009) as docking engine with default parameters. Graphical representations of the docking results as PyMOL (http://www.pymol.org) sessions were downloaded from BDS with default options as specified on the website.

All the protocols comply with the recommendation, the approval of which was obtained from the participant institutions and in accordance to the ethical standards laid down in the 1964 Declaration of Helsinki and its later amendments.

\section{Cell culture}

Distinct human colorectal adenocarcinoma cell lines HCT-116, DLD-1, SW480, HCT15, HT-29, LS174T, SW620 and LoVo were obtained from the American Type Culture Collection (ATCC, Rockville, MD, USA). Cell lines were cultivated using standard high glucose Dulbecco's Modified Eagle's Medium (DMEM) supplemented with $10 \%$ heat-inactivated fetal bovine serum (FBS), $50 \mathrm{U} / \mathrm{mL}$ penicillin and $50 \mu \mathrm{g} / \mathrm{mL}$ streptomycin (all from Sigma Aldrich Chemical Co., USA) at $37{ }^{\circ} \mathrm{C}$ and $5 \% \mathrm{CO}_{2}$ and 95\% humidified atmosphere. Subculturing was performed when cells reached $90 \%$ confluence. Cell RNA extraction and qPCR for Fascin1 expression quantification is described in Supplementary Material S1. The human colorectal carcinoma cells were 
genetically overexpressed (DLD-1) and knocked-down (HCT-116) for Fascin-1 (Supplementary Material S1).

\section{Cell viability assay}

Exponentially growing cells were plated in flat-bottomed 96-well plates (Nunc, Roskilde, Denmark) in triplicate (1500 cells/well). Cells were treated with a series of concentrations from $500 \mathrm{nM}$ to $100 \mu \mathrm{M}$ of either migrastatin or compound $\mathrm{G} 2$ up to 3 days (24, 48 and 72 hours) in a $5 \% \mathrm{CO}_{2}$ humidified atmosphere. Control cells were treated with drug carrier [0.1\% dimethyl sulfoxide (DMSO)]. Cells were assayed for viability as follows. Briefly, Dulbecco's phosphate-buffered saline (DPBS) supplemented with $1.9 \mathrm{mg} / \mathrm{mL}$ tetrazolium (MTT) $\mathrm{pH} 7.2$ was added to the cells (30 $\mu \mathrm{L} /$ well). After incubation at $37^{\circ} \mathrm{C}$ for 4 hours, the medium was carefully aspirated. The formazan crystals were dissolved in $200 \mu \mathrm{L}$ DMSO for 30 min and the absorbance was read in a microtiter plate reader at $570 \mathrm{~nm}$ and $620 \mathrm{~nm}$ as reference. Results were calculated as: cell viability $(\%)=$ average optical density (O.D.) of wells/average O.D. of control wells.

\section{Cell Migration Assay}

Cell migration was studied using HCT-116 and DLD-1 cell lines by performing a scratch wound healing assay in standard medium supplemented with 5\% FBS. Typically, 50,000 cells were plated in low 35-mm-dishes with culture inserts following manufacturer instructions (Ibidi, Martinsried, Germany). After appropriate cell attachment and monolayer formation (around 24 hours), inserts were removed with 
sterile forceps to create a wound field of approximately $500 \mu \mathrm{m}$. Detached cells were gently removed with DPBS before the addition of drugs. Confluent cells were incubated in one of the following treatments: control (0.1\% DMSO), $100 \mu \mathrm{M}$ migrastatin, 5,10 and $20 \mu \mathrm{M}$ compound G2. Cells were then placed in a cell culture incubator and they were allowed to migrate. At 0,4 and 7 hours (linear growth phase), 10 fields of the injury area were photographed with an inverted phase contrast microscope using 10X magnification. For each time point, the area uncovered by cells was determined by Image $\mathbf{J}$ software (National Institute of Health, Bethesda, MD, USA). Each treatment was performed in triplicate.

The migration speed of the wound closure was given as the percentage of the recovered area at each time point, relative to the initially covered area $\left(\mathrm{t}_{0}\right)$. The velocity of wound closure $(\% / \mathrm{h})$ was calculated according to the formula:

$$
\text { Slope }(\% / \mathrm{h})=\frac{\left(\% \text { covered area } t_{x}\right)-\left(\% \text { covered area } t_{o}\right)}{\left(t_{x}-t_{o}\right)}
$$

Slopes are expressed as percentages relative to control conditions.

\section{Transwell invasion assay}

The invasive capacity of HCT-116 cells was determined using Cytoselect TM 24 Well Cell Invasion Assay (Basement Membrane Colorimetric Format) with Matrigel $^{\mathrm{R}}$ coated Transwell chambers ( $8 \mu \mathrm{m}$ pore size) (Cell Biolabs Inc., CA, USA). Briefly, cells $\left(9.5 \times 10^{4}\right)$ were resuspended in serum-free medium with corresponding inhibitors $(100$ $\mu \mathrm{M}$ migrastatin and $20 \mu \mathrm{M}$ compound $\mathrm{G} 2$ ) and seeded into the upper chamber. Additionally, $500 \mu \mathrm{L}$ of standard medium were added to the well. After 30 hours of incubation, cells that remained on the upper chamber were scraped away with a cotton 
swab, and the cells that had migrated through the matrigel and reached the bottom of the chamber were stained with the cell stain solution provided in the kit. Invasiveness was quantified by counting cells on the lower surface of the filter using Image $\mathbf{J}$ software (National Institute of Health, Bethesda, MD, USA). In addition, invasive cells at the bottom side of the filter were eluted and quantified at an absorbance of $560 \mathrm{~nm}$.

\section{Myoma organotypic invasion model}

Tumoral cell invasion was assessed in the myoma organotypic cultures and performed according to the previously published myoma model protocol (Nurmenniemi et al., 2009; Åström, Heljasvaara, Nyberg, Al-Samadi, \& Salo, 2018). Briefly, uterine leiomyoma tissues were obtained from routine surgery after informed consent of the donors and their use approved by The Ethics Committee of the Oulu University Hospital. The myoma tissue was sliced into $5 \mathrm{~mm}$ and disks were made with an $8-\mathrm{mm}$ biopsy punch (Kai Industries Co., Gifu, Japan). Myoma disks were pre-incubated in either $0.1 \%$ DMSO, $100 \mu \mathrm{M}$ migrastatin, $10 \mu \mathrm{M}$ and $20 \mu \mathrm{M}$ compound $\mathrm{G} 2$ at $4{ }^{\circ} \mathrm{C}$ for 48 hours. The myoma disks were placed into Transwell inserts (diameter $6.5 \mathrm{~mm}$; Corning Incorporated, Corning, NY) and 700,000 cells in $50 \mu \mathrm{L}$ of media were added on top of each myoma disk. Cells were allowed to attach overnight and the myoma disks, transferred onto uncoated nylon disks and be treated with the compounds for 14 days, while changing the treatment media every 3 days. Subsequently the myoma discs were fixed with $4 \%$ neutral buffered formalin for 24 hours and $6 \mu \mathrm{m}$ sections were cut and stained with cytokeratin AE1/AE3 (M3515, Dako). Sections were documented at 10X magnifications, using the Leica DMRB microscope DFC 480 camera with the Leica application suite v3.8 (Leica Microsystems, Wetzlar, Germany). Image J software 
(National Institute of Health, Bethesda, MD, USA) was used to measure invasion areas and depths. Each treatment was performed in triplicate.

\section{Zebrafish invasion assays and treatments}

The colonization of zebrafish (ZF) (Danio rerio) embryos by human cancer colorectal cells lines was performed as previously described (Jelassi et al., 2011). The experiments performed comply with the Guidelines of the European Union Council (Directive 2010/63/EU) and the Spanish RD 53/2013. Experiments and procedures were performed as approved by the Bioethical Committee of the Murcia University (Spain). Briefly, human cell lines were trypsinized, washed and stained with fluorescent CM-Dil (Vibrant, Invitrogen) following manufacturer's instructions. Fifty to 100 labelled cells were injected into the yolk sac of dechorionated ZF embryos and transferred into 24 well plates. Fish with fluorescently labelled cells appearing outside the implantation area at 2 hours post-injection (dpi) were excluded from further analysis. All other fishes were treated, by bath immersion, with $\mathrm{E} 3$ medium $(5 \mathrm{mM} \mathrm{NaCl}, 0.33 \mathrm{mM} \mathrm{KCl}, 0.33$ $\mathrm{mM} \mathrm{CaCl}_{2}, 0.33 \mathrm{mM} \mathrm{MgSO}_{4}, 0.1 \%$ methylene blue) (all from Sigma-Aldrich, St Louis, MO, USA) supplemented with either $100 \mu \mathrm{M}$ migrastatin or 5-10 $\mu \mathrm{M}$ compound $\mathrm{G} 2$ for 96 hours at $35{ }^{\circ} \mathrm{C}$. Treatment was renewed every 24 hours. Larvae analyzed with a M205-FA stereomicroscope equipped with a DFC365FX camera (Leica). The evaluation criteria for embryos being colonized by human cancer cells was the presence of more than three cells outside of the yolk sac and, with this criterion, ZF percentage of invasion was calculated.

With the aim of finding out whether the pro-metastatic activity of Fascin1-induced expression was affected by chemical compounds, a metastasis assay was carried out. 
Zebrafish embryos xenografted with Fascin1-transfected DLD-1 cells were incubated up to 144 hours. The day of invasion screening (fourth day post xenograft), freshly prepared E3 medium was supplemented with $100 \mu \mathrm{M}$ migrastatin or $10 \mu \mathrm{M}$ compound G2 together with 375 rotifers/mL (Brachionus plicatilis (L-type); ReefNutrition, Campbell, CA, USA). The number of small colonies generated from individual invading cells was counted on the sixth day post xenograft (6 dpi). This was considered as the number of ZF larvae with metastasis.

\section{Data analysis}

Data are expressed as mean \pm standard deviation $(\mathrm{SD})$. Data were analyzed for statistical differences by the Student's t-test for paired and unpaired data after testing for normal distribution of the data. For in vitro experiments, one-way analysis of variance (ANOVA) was performed followed by a Tukey poshoc test to compare each group. Differences were considered significant at an error probability of $\mathrm{P}<0.05$. SPSS 18.0 software was used for the rest of statistical analyses (SPSS, Inc, Chicago, Illinois, USA). For myoma assay OriginPro 2016 software was used for statistical calculations. One-way ANOVA (analysis of variance) with post-hoc comparisons based on the Tukey's multiple comparisons test were applied. The level of significance considered was $5 \%(\mathrm{p} \leq 0.05)$.

\section{Results \\ Molecular Modeling}

Figure 1 shows the main resulting pose of compound G2 after blind docking calculations against whole Fascin1 protein surface. Most relevant intermolecular 
interactions established are related to hydrogen bonds (GLU215), pi-pi (TRP101) and hydrophobic interactions (LEU16, PHE14, LEU48, ILE93, VAL134, GLU215, PHE216). It must be noticed that these residues participate in the actin-binding site 2 , located within the pocket formed by $\beta$-trefoil 1 and 2 from the Fascin 1 structure, and that blind docking calculations were able to predict this interaction spot with no previous assumption about binding site. In addition, this prediction coincides rather well with crystallographic pose of ligand compound G2-029 (PDB: 6B0T), which is to be expected due to the small difference between compound G2 and G2-029 molecules, just a methyl group.

\section{Compound $G 2$ prevents in vitro Fascin1-induced F-actin bundling}

In order to assess the effect of compound G2 on Fascin1, we performed an F-actin bundling assay under transmission electron microscopy (TEM) (Supplementary material S1). As shown in Figure 2, only F-actin incubated in the presence of untreated Fascin1 formed filament bundles (12.00 [9.00-19.75]). Fascin1 preincubated with $100 \mu \mathrm{M}$ migrastatin or compound G2 (up to $20 \mu \mathrm{M}$ ) lead to the disorganization of the bundles, resulting in fewer filaments than in control conditions (Kruskal-Wallis test, $\mathrm{p}<0.001$ ). No statistically changes were found between the different drug treatments (KruskalWallis test, $\mathrm{p}=0.370)$.

Compound G2 affects cytoskeleton formation and Fascinl localization at the lamellipodium 
In order to choose colorectal cell lines with highest and lowest endogenous Fascin1 expression, a RT-qPCR was performed upon RNA extracted from eight cell lines. As shown in Supplementary Material 2, HCT-116 and SW480 exhibited the highest Fascin1 expression whilst LoVo, DLD-1 and HT-29 had the lowest. Given the ease for cell culture and the differences in endogenous Fascin1 expression, we selected DLD-1 (low Fascin1 expression) and HCT-116 (high Fascin1 expression) cell lines in subsequent assays.

To assess the Fascin 1 inhibition activity of the compound G2 on cell viability, we tested different concentration of migrastatin and compound G2 on different colorectal cancer cells. As shown Supplementary Material S3, migrastatin was generally better tolerated than compound G2 by the DLD-1 and HCT-116 cell lines. Thus, the working concentrations for migrastatin and compound G2 were set up for subsequent in vitro studies at $100 \mu \mathrm{M}$ and up to $20 \mu \mathrm{M}$, respectively.

The effect of compound G2 on Fascin1 localization and the reorganization of the actin cytoskeleton as well as on the protrusion of lamellipodium at the cell front was assessed by immunofluorescence. For that purpose, we used HCT-116, the colorectal cell line with maximum expression levels of Fascin1 together with Fascin1 silenced HCT-116 cells. As shown in Figure 3, prominent lamellipodium formation was observed in control conditions and for EGF treated cells (non-significant differences between each other were found). However, these cytoskeleton structures were absent in cells treated with migrastatin and compound G2, similarly to what it was observed with PD98059, an inhibitor targeting the MEK pathway. Lamellipodium protrusion numbers calculated at different conditions were significantly lower upon both migrastatin and compound G2 treatments when compared to control conditions (Table 1). Supplementary material S4 
showed that the treatments also abolished the actin bundles in lamellipodium. Similar results were observed with an extra cell line expressing Fascin1 (HaCat) as DLD1

morphology was not suitable for assessing lamellipodium formation (data not shown).

Transcriptional Fascin1 silencing of HCT-116 abolished the Fascin1 accumulation at

lamellipodium sites and produced an alteration of F-actin microfilament assembly in

silenced cells (Supplemental S5D).

Compound G2 diminishes migration and inhibits Matrigel cell invasion of colorectal cancer cells

In order to correlate the observed effect of Fascin1 inhibitors on lamellipodium protrusion to an effect on cell migration, migrastatin and compound G2 treated cells were investigated for their migration activity using an in vitro wound healing scratch assay. As shown in Figure 4, compound G2 produces a remarkable inhibition of migration in HCT-116 and DLD-1 cell lines $(\mathrm{p}<0.01)$. Compound G2 effect at $20 \mu \mathrm{M}$ was more pronounced than migrastatin in DLD-1 cells, whist in HCT-116 the effect of compound $\mathrm{G} 2$ on migration was lower.

Tumor cell invasion not only involves the acquisition of migration properties but also the ability to degrade the basement membrane and tumor stoma matrices, threedimensional substrates (Albini, 1998).For that reason, we performed a Transwell assay on Matrigel $^{\mathrm{R}}$ which resembles the basement membrane extracellular matrix composition. As shown in Supplementary material S6, both compound G2 and migrastatin inhibit tumor cell invasion of HCT-116, the effect of the latter being slightly more evident.

To further confirm the inhibitory effect of compound G2 on the Fascin1 activity, we used Fascin1 silenced HCT116 and fascin1 overexpressed DLD-1 cells and tested their 
migration and invasion properties. Fascin1 silencing produces a slight decrease of migration and invasion compared to MOCK HCT-116 cells $(\mathrm{p}<0.05)$, whereas double inhibition (genetic and pharmacological) only produced a significant decrease in migration (Supplemental material S7A-B). Accordingly, $10 \mu \mathrm{M}$ compound G2 strongly diminished migration and invasion in Fascin1 overexpressed DLD-1 cells $(p<0.01)$ (Supplemental material S7C-D).

Compound G2 diminishes HCT-116 colon cancer cells invasion in a human benign leiomyoma tissue in vitro $3 D$ model

In order to find out whether the anti-invasive properties of compound G2 on cancer cell invasion could be translated into a 3D human model, a myoma disc organotypic model was used. As shown in Figure 5, compound G2 $(5 \mu \mathrm{M})$ significantly reduced the invasion depth and invasion area of HCT-116 cells into the discs when compared to untreated cells $(\mathrm{P}<0.01)$. Similar results were obtained when cells were treated with 10 and $20 \mu \mathrm{M}$ compound G2 (Supplementary material S8).

Compound G2 inhibits the invasive capacity of HCT-116 tumor cells in an in vivo assay of zebrafish model

In order to find out whether the anti-invasive properties of compound G2 could be extrapolated to a living animal, a xenograft assay was carried by using the wellestablished zebrafish (ZF)-larvae invasion model. To test viability, groups of thirty larvae were treated with either $100 \mu \mathrm{M}$ migrastatin or 5, 10 and $20 \mu \mathrm{M}$ compound $\mathrm{G} 2$. The majority of larvae were viable after three days treatment with either 5 or $10 \mu \mathrm{M}$. However, $20 \mu \mathrm{M}$ compound G2 treatment caused larvae death at the third day of treatment (Supplementary material S9A). Therefore, concentrations chosen for ZF 
assays were 5 and $10 \mu \mathrm{M}$ compound G2. Later on, detached and labelled colorectal cancer cells were injected into the yolk sac and colonization was followed up to 6 days. The percentage of invasion was studied for four colorectal cancer cell lines (Fig. 6A and 6B) that exhibited different Fascin1 expression levels (Supplementary material S2). HCT-116 and DLD-1 were selected for being the cell lines with the highest and lower Fascin1 expression, respectively. Good correlation was observed between Fascin1 expression and cell invasion for all cell lines assayed except HCT-15 whose invasion was lower than expected (Figure 6B) possibly due to other fascin1 independent factors affecting invasion (Stevenson, Veltman, \& Machesky, 2012). Therefore, and because of good correlation, HCT-116 was selected for being the cell line with the highest and DLD-1 for its lower Fascin1 expression and cell invasion. To further test the in vivo involvement of Fascin1 in this phenomenon, we performed exogenous silencing of Fascin1 in HCT-116 cells (Figure 7A). Either genetic reduction of Fascin1 or its pharmacological inhibition by compound G2 decreased the invasion in HCT-116 cells (Figures 7B and 7C). Conversely, we induced the over-expression of Fascin1 by means of plasmid transfection into DLD-1 cells (Figure 7D), thus correlating with a higher invasion capacity (Figure 7E). Compound G2 kept inhibiting invasion in both, MOCK and transfected Fascin1-overexpressing cells (Figure 7F). Consequently, in vivo invasion correlated with Fascin1 expression and compound G2 inhibited it in transfected cells (Figures 7E and 7F). Furthermore, a clear increase of the number of ZF larvae with metastasis was observed with Fascin1-overexpressing DLD-1 cells although $10 \mu \mathrm{M}$ compound G2 treatment had a similar effect on ZF groups subjected to either Fascin1 transfected or wild-type DLD-1 cells (Supplementary material S10).

\section{Discussion}


Colorectal serrated adenocarcinoma and triple negative breast carcinoma are characterized by their bad prognosis, over-expression of Fascin 1 and the absence of targeted molecular therapy (Conesa-Zamora et al., 2013; Esnakula et al., 2014; GarcíaSolano, Conesa-Zamora, Carbonell, et al., 2012; García-Solano et al., 2016; Ghebeh et al., 2014; Kanda et al., 2014; Rodríguez-Pinilla et al., 2006; Stefanius et al., 2011; Wang et al., 2016). Han et al. demonstrated an inhibitory effect of compound G2 and its derivates on Fascin1-driven actin bundling. Moreover, they showed an anti-migratory and anti-invasive effect of compound G2 on breast cancer cells (Han et al., 2016). In the present report we include a molecular model, which provided an improved understanding regarding atomic details of the interactions between compound G2 chemical class and Fascin1 inhibition and will guide the future identification of more potent anti-metastatic drugs. Using blind docking calculations, the model identified a region in Fascin1 possibly involved in compound G2 binding. Some prior evidences support this finding as this region is part of the actin binding site 1, as described by Han et al. (Han et al., 2016). In accordance, preincubation of Fascin 1 with migrastatin and compound G2 disrupted the F-actin bundle formation in vitro. Herein, among the different colorectal cell lines used in this study, HCT-116 expressed the highest Fascin 1 levels and its migration capacity was clearly reduced after treatment with compound G2, even at lower concentrations than migrastatin and in a dose-dependent manner. Invasion and confocal studies were further performed with HCT-116 cells because of its morphological features and higher Fascin1 expression levels. We showed that both inhibitors, migrastatin and compound G2, strongly abolished the protrusion of lamellipodium, as confirmed by rescue experiments in Fascin1 silenced cells. Furthermore, we observed similar inhibitory effects of migrastatin and compound G2 on the Matrigel invasion assays with naïve and transfected HCT-116 and DLD-1 cells. 
Myoma discs represent a superior 3D model for cancer cell invasion studies compared assay, both migrastatin and compound G2 were similarly able to significantly decrease both the invasion depth and invasion area of HCT-116 cells. It is important to notice that cell death caused by long-time incubation with the compound G2 was avoided in the migration and invasion in vitro assays, since viability did not seem to be compromised after 30 hours. Of note, no previous studies have analyzed the effect of migrastatin or compound G2 on human solid, hypoxic, myoma disc organotypic invasion assay.

\section{Moreover, in vivo assays using a model of ZF where Fascin1 expression was modified} in colorectal cells showed that compound G2 effects on colorectal cancer cells invasion capacities showed a Fascin1 dependency. This compound inhibits the invasion of two cell lines of colorectal cancer with high expression of Fascin1, both constitutive and induced, without affecting the ZF viability and with a more pronounced effect than migrastatin. This activity also had an effect on colony formation from individual invading cells thus suggesting an inhibition of both invasion and metastasis of Fascin1tranfected tumor cells. It is worth of mentioning that this validated model was capable of testing a very high number of individuals per assay. However and despite these evidences, additional off-target anti-tumoral effects of G2 apart from Fascin1 are also possible.

In conclusion, this study reports a Fascin1 inhibitor with anti-migratory and antiinvasive properties in colorectal cancer cells at lower concentrations than migrastatin, the typical Fascin1 inhibitor. Using this strategy, we have here confirmed a novel class of compounds for the study of therapeutic approaches for invasive and metastatic 
tumoral cells, such as SAC; providing the first rationale for a tailored therapy in this

type of cancer.

\section{REFERENCES}

Albini, A. (1998). Tumor and endothelial cell invasion of basement membranes. The matrigel chemoinvasion assay as a tool for dissecting molecular mechanisms. Pathol Oncol Res, 4(3), 230-241. doi:10.1007/bf02905254

Cao, H. H., Zheng, C. P., Wang, S. H., Wu, J. Y., Shen, J. H., Xu, X. E., . . Xu, L. Y. (2014). A molecular prognostic model predicts esophageal squamous cell carcinoma prognosis. PLoS One, 9(8), e106007. doi:10.1371/journal.pone.0106007

Chen, L., Yang, S., Jakoncic, J., Zhang, J. J., \& Huang, X. Y. (2010). Migrastatin analogues target fascin to block tumour metastasis. Nature, 464(7291), 1062-1066. doi:10.1038/nature08978

Conesa-Zamora, P., García-Solano, J., García-García, F., Turpin, M. e. C., Trujillo-Santos, J., Torres-Moreno, D., . . . Pérez-Guillermo, M. (2013). Expression profiling shows differential molecular pathways and provides potential new diagnostic biomarkers for colorectal serrated adenocarcinoma. Int J Cancer, 132(2), 297-307. doi:10.1002/ijc.27674

Esnakula, A. K., Ricks-Santi, L., Kwagyan, J., Kanaan, Y. M., DeWitty, R. L., Wilson, L. L., . . . Naab, T. J. (2014). Strong association of fascin expression with triple negative breast cancer and basal-like phenotype in African-American women. J Clin Pathol, 67(2), 153160. doi:10.1136/jclinpath-2013-201698

García-Solano, J., Conesa-Zamora, P., Carbonell, P., Trujillo-Santos, J., Torres-Moreno D, D., Pagán-Gómez, I., . . Pérez-Guillermo, M. (2012). Colorectal serrated adenocarcinoma shows a different profile of oncogene mutations, MSI status and DNA repair protein expression compared to conventional and sporadic MSI-H carcinomas. Int J Cancer, 131(8), 1790-1799. doi:10.1002/ijc.27454

García-Solano, J., Conesa-Zamora, P., Trujillo-Santos, J., Mäkinen, M. J., \& Pérez-Guillermo, M. (2011). Tumour budding and other prognostic pathological features at invasive margins in serrated colorectal adenocarcinoma: a comparative study with conventional carcinoma. Histopathology, 59(6), 1046-1056. doi:10.1111/j.13652559.2011.04043.x

García-Solano, J., Conesa-Zamora, P., Trujillo-Santos, J., Torres-Moreno, D., Mäkinen, M. J., \& Pérez-Guillermo, M. (2012). Immunohistochemical expression profile of $\beta$-catenin, Ecadherin, P-cadherin, laminin-5ץ2 chain, and SMAD4 in colorectal serrated adenocarcinoma. Hum Pathol, 43(7), 1094-1102. doi:10.1016/j.humpath.2011.08.020

García-Solano, J., García-Solano, M. E., Torres-Moreno, D., Carbonell, P., Trujillo-Santos, J., Pérez-Guillermo, M., \& Conesa-Zamora, P. (2016). Biomarkers for the identification of precursor polyps of colorectal serrated adenocarcinomas. Cell Oncol (Dordr), 39(3), 243-252. doi:10.1007/s13402-016-0269-5

García-Solano, J., Pérez-Guillermo, M., Conesa-Zamora, P., Acosta-Ortega, J., Trujillo-Santos, J., Cerezuela-Fuentes, P., \& Mäkinen, M. J. (2010). Clinicopathologic study of 85 colorectal serrated adenocarcinomas: further insights into the full recognition of a new subset of colorectal carcinoma. Hum Pathol, 41(10), 1359-1368. doi:10.1016/j.humpath.2010.04.002

Gasteiger, J., \& Marsili, M. (1980). Iterative partial equalization of orbital electronegativity-a rapid access to atomic charges Y1 - 1980 Y2 - 1980. Tetrahedron, 36(22), 3219-3228. doi:https://doi.org/10.1016/0040-4020(80)80168-2 M1 - Generic 
Gaul, C., Njardarson, J. T., Shan, D., Dorn, D. C., Wu, K. D., Tong, W. P., . . Danishefsky, S. J. (2004). The migrastatin family: discovery of potent cell migration inhibitors by chemical synthesis. J Am Chem Soc, 126(36), 11326-11337. doi:10.1021/ja048779q

Ghebeh, H., Al-Khaldi, S., Olabi, S., Al-Dhfyan, A., Al-Mohanna, F., Barnawi, R., . . Al-Alwan, M. (2014). Fascin is involved in the chemotherapeutic resistance of breast cancer cells predominantly via the PI3K/Akt pathway. $\mathrm{Br} J$ Cancer, 111(8), 1552-1561. doi:10.1038/bjc.2014.453

Han, S., Huang, J., Liu, B., Xing, B., Bordeleau, F., Reinhart-King, C. A., . . Huang, X. Y. (2016). Improving fascin inhibitors to block tumor cell migration and metastasis. Mol Oncol, 10(7), 966-980. doi:10.1016/j.molonc.2016.03.006

Hashimoto, Y., Kim, D. J., \& Adams, J. C. (2011). The roles of fascins in health and disease. J Pathol, 224(3), 289-300. doi:10.1002/path.2894

Hashimoto, Y., Skacel, M., \& Adams, J. C. (2005). Roles of fascin in human carcinoma motility and signaling: prospects for a novel biomarker? Int I Biochem Cell Biol, 37(9), 17871804. doi:10.1016/j.biocel.2005.05.004

Huang, J., Dey, R., Wang, Y., Jakoncic, J., Kurinov, I., \& Huang, X. Y. (2018). Structural Insights into the Induced-fit Inhibition of Fascin by a Small-Molecule Inhibitor. J Mol Biol, 430(9), 1324-1335. doi:10.1016/j.jmb.2018.03.009

Jelassi, B., Chantôme, A., Alcaraz-Pérez, F., Baroja-Mazo, A., Cayuela, M. L., Pelegrin, P., . . . Roger, S. (2011). P2X(7) receptor activation enhances SK3 channels- and cystein cathepsin-dependent cancer cells invasiveness. Oncogene, 30(18), 2108-2122. doi:10.1038/onc.2010.593

Jones, R. P., Bird, N. T., Smith, R. A., Palmer, D. H., Fenwick, S. W., Poston, G. J., \& Malik, H. Z. (2015). Prognostic molecular markers in resected extrahepatic biliary tract cancers; a systematic review and meta-analysis of immunohistochemically detected biomarkers. Biomark Med, 9(8), 763-775. doi:10.2217/BMM.15.48

Kanda, Y., Kawaguchi, T., Kuramitsu, Y., Kitagawa, T., Kobayashi, T., Takahashi, N., . . . Okada, F. (2014). Fascin regulates chronic inflammation-related human colon carcinogenesis by inhibiting cell anoikis. Proteomics, 14(9), 1031-1041. doi:10.1002/pmic.201300414

Li, A., Morton, J. P., Ma, Y., Karim, S. A., Zhou, Y., Faller, W. J., . . Machesky, L. M. (2014). Fascin is regulated by slug, promotes progression of pancreatic cancer in mice, and is associated with patient outcomes. Gastroenterology, 146(5), 1386-1396.e1381-1317. doi:10.1053/j.gastro.2014.01.046

Machesky, L. M., \& Li, A. (2010). Fascin: Invasive filopodia promoting metastasis. Commun Integr Biol, 3(3), 263-270. doi:10.4161/cib.3.3.11556

Morris, G. M., Huey, R., Lindstrom, W., Sanner, M. F., Belew, R. K., Goodsell, D. S., \& Olson, A. J. (2009). AutoDock4 and AutoDockTools4: Automated docking with selective receptor flexibility. J Comput Chem, 30(16), 2785-2791. doi:10.1002/jcc.21256

Nurmenniemi, S., Sinikumpu, T., Alahuhta, I., Salo, S., Sutinen, M., Santala, M., . . Salo, T. (2009). A novel organotypic model mimics the tumor microenvironment. Am J Pathol, 175(3), 1281-1291. doi:10.2353/ajpath.2009.081110

Omran, O. M., \& Al Sheeha, M. (2015). Cytoskeletal Focal Adhesion Proteins Fascin-1 and Paxillin Are Predictors of Malignant Progression and Poor Prognosis in Human Breast Cancer. J Environ Pathol Toxicol Oncol, 34(3), 201-212.

Rodrigues, P. C., Sawazaki-Calone, I., Ervolino de Oliveira, C., Soares Macedo, C. C., Dourado, M. R., Cervigne, N. K., . . Coletta, R. D. (2017). Fascin promotes migration and invasion and is a prognostic marker for oral squamous cell carcinoma. Oncotarget, 8(43), 74736-74754. doi:10.18632/oncotarget.20360

Rodríguez-Pinilla, S. M., Sarrió, D., Honrado, E., Hardisson, D., Calero, F., Benitez, J., \& Palacios, J. (2006). Prognostic significance of basal-like phenotype and fascin expression in node-negative invasive breast carcinomas. Clin Cancer Res, 12(5), 1533-1539. doi:10.1158/1078-0432.CCR-05-2281 
Salo, T., Dourado, M. R., Sundquist, E., Apu, E. H., Alahuhta, I., Tuomainen, K., . . Al-Samadi, A. (2018). Organotypic three-dimensional assays based on human leiomyoma-derived matrices. Philos Trans R Soc Lond B Biol Sci, 373(1737). doi:10.1098/rstb.2016.0482

Stefanius, K., Ylitalo, L., Tuomisto, A., Kuivila, R., Kantola, T., Sirniö, P., . . . Mäkinen, M. J. (2011). Frequent mutations of KRAS in addition to BRAF in colorectal serrated adenocarcinoma. Histopathology, 58(5), 679-692. doi:10.1111/j.13652559.2011.03821.x

Stevenson, R. P., Veltman, D., \& Machesky, L. M. (2012). Actin-bundling proteins in cancer progression at a glance. J Cell Sci, 125(Pt 5), 1073-1079. doi:10.1242/jcs.093799

Stewart, C. J., \& Crook, M. L. (2015). Fascin expression in undifferentiated and dedifferentiated endometrial carcinoma. Hum Pathol, 46(10), 1514-1520. doi:10.1016/j.humpath.2015.06.011

Sánchez-Linares, I., Pérez-Sánchez, H., Cecilia, J. M., \& García, J. M. (2012). High-Throughput parallel blind Virtual Screening using BINDSURF. BMC Bioinformatics, 13 Suppl 14, S13. doi:10.1186/1471-2105-13-S14-S13

Tan, V. Y., Lewis, S. J., Adams, J. C., \& Martin, R. M. (2013). Association of fascin-1 with mortality, disease progression and metastasis in carcinomas: a systematic review and meta-analysis. BMC Med, 11, 52. doi:10.1186/1741-7015-11-52

Wang, C. Q., Tang, C. H., Chang, H. T., Li, X. N., Zhao, Y. M., Su, C. M., . . Huang, B. F. (2016). Fascin-1 as a novel diagnostic marker of triple-negative breast cancer. Cancer Med, 5(8), 1983-1988. doi:10.1002/cam4.746

Zhao, W., Gao, J., Wu, J., Liu, Q. H., Wang, Z. G., Li, H. L., \& Xing, L. H. (2015). Expression of Fascin-1 on human lung cancer and paracarcinoma tissue and its relation to clinicopathological characteristics in patients with lung cancer. Onco Targets Ther, 8, 2571-2576. doi:10.2147/OTT.S81915

Åström, P., Heljasvaara, R., Nyberg, P., Al-Samadi, A., \& Salo, T. (2018). Human Tumor TissueBased 3D In Vitro Invasion Assays. Methods Mol Biol, 1731, 213-221. doi:10.1007/9781-4939-7595-2_19 
Table 1. Lamellipodium protrusion numbers in the different conditions in HCT-116 cells.

\begin{tabular}{lccccc} 
& Control & $100 \mu \mathrm{M}$ & $20 \mu \mathrm{M}$ & $10 \mathrm{ng} / \mathrm{mL}$ & $50 \mu \mathrm{M}$ \\
& & migrastatin & compound G2 & EGF & PD98059 \\
& & & & & \\
\hline Lamellipodium & $9 \pm 1.5$ & $2 \pm 2$ & $5.8 \pm 1.1$ & $10.4 \pm 1.5$ & $1 \pm 1$ \\
numbers & & & & \\
\hline P value* & $\mathbf{0 , 0 0 0 1 3 9}$ & $\mathbf{0 , 0 0 2 9 3 6}$ & 0,140178 & $\mathbf{5 , 9 2 4 1 E}$ \\
& & & & \\
\hline
\end{tabular}




\section{Figure legends}

Figure 1. Main interactions obtained after blind docking of compound G2 against Fascin1. Blue solid line represents hydrogen bond, green dashed line aromatic interactions, and dashed grey lines hydrophobic interactions. Inset: compound G2 chemical structure.

Figure 2. Transmission electronic microscopy visualization of actin binding and bundling activities of Fascin1 treated or not with inhibitors (negative staining). A) Stained F-actin filaments alone. B) F-actin bundling assay with filamentous F-actin and untreated Fascin1 (1:1 molecular ratio) (control condition). C) F-actin bundling assay with filamentous F-actin and Fascin1 previously incubated with $100 \mu \mathrm{M}$ migrastatin (1:1 molecular ratio). D) F-actin bundling assay with filamentous F-actin and Fascin1 previously incubated with $20 \mu \mathrm{M}$ compound G2. Magnification: A $120.000 \mathrm{X}$ and B to D 93.000X. E) Quantitative analysis of the numbers of actin filaments bundles in the stated conditions (*** $\mathrm{p}<0.001$ compared to control condition), non-significant (N.S.) between treatments (Kruskal-Wallis test).

Figure 3. Migrastatin and compound G2 affect lamellipodium formation and morphology of HCT-116. Representative images of immunofluorescence assays for Fascin1 (lower inset in detail) are shown. A) Control condition; B) $100 \mu \mathrm{M}$ migrastatin; C) $20 \mu \mathrm{M}$ compound G2; D) $50 \mu \mathrm{M}$ PD98059 (Mek inhibitor); E) $10 \mathrm{ng} / \mathrm{mL}$ Epidermal Growth Factor (EGF, migration stimulator). Cells were fixed and stained with antiFascin1 antibody (1/250). Images were captured with a LSM 510 META confocal fluorescence microscope with $63 \mathrm{X}$ oil objective. Fascin 1 location is show in green in these structures. Co-staining with Hoechst-33258 was used to show the cell nuclei. Both 
migrastatin and compound G2 inhibited lamellipodium protrusion and Fascin1 localization in a similar way to the migration Mek inhibitor.

Figure 4. Migrastatin and compound G2 prevent cell migration on HCT-116 and DLD1 colorectal cancer cells. A) Effect of $100 \mu \mathrm{M}$ migrastatin and $20 \mu \mathrm{M}$ compound $\mathrm{G} 2$ on HCT-116 and B) DLD-1 cells. C) Percentage of migration for $100 \mu \mathrm{M}$ migrastatin and compound $\mathrm{G} 2(5,10,20 \mu \mathrm{M})$ treatments. Migration was calculated with respect to the control conditions $(100 \%)$ for a slope between 4 and 7 hours (lineal phase). $* * p<0.01$.

Figure 5. Myoma organotypic invasion model in HCT-116 cell line. A) Invasion of the cells within myoma discs after treatment in control conditions, B) $100 \mu \mathrm{M}$ migrastatin, and C) $5 \mu \mathrm{M}$ compound G2. Pictures were taken under a phase contrast microscope with 100X magnification and $100 \mu \mathrm{m}$ scale bars. D) The invasion area and E) Invasion depth of cytokeratin positive cells were quantified with image J. Data is shown as mean \pm SD compared with the control. ***p $<0.001$

Figure 6. Zebrafish invasion assays. A) Zebrafish invasion model. Representative images of a zebrafish embryo with no invasion where the labeled cells remained in the yolk and never invaded the embryo and an embryo with invasion where the cells were able to migrate outside of the yolk. Magnification of the tail region of an embryo with invasion is shown at the right. B) Invasiveness of each cell line in this model. Data is shown as mean $\pm \mathrm{SD}$; compared with the control. * $\mathrm{p}=0,049-0,01 ; * * \mathrm{p}=0,001-0,009$.

Figure 7. Treatment effects on cancer cell invasion with different grades of Fascin1 expression. A) Inhibition of Fascin1 gene expression upon siRNA-Fascin1 transfection in HCT-116 cells. B) Effect of 5 and $10 \mu \mathrm{M}$ compound G2 on HCT-116 invasion with endogenous and silenced Fascin1 expression. C) Compound G2 inhibitory activity on control and siRNA Fascin1-transfected HCT-116 cells. First two columns represent the 
difference between percentage of invasion in control (MOCK) and percentage of invasion in treated cells. Second two columns represent the difference between percentage of invasion in siRNA Fascin1-transfected HCT-116 and percentage of invasion in compound G2-treated siRNA Fascin1-transfected HCT-116 cells. Note that the effect of compound G2 decreased when Fascin1 was silenced. D) Overexpression of Fascin1 gene upon Fascin1 transfection in DLD-1. E) Effect of 5 and $10 \mu \mathrm{M}$ compound G2 on DLD-1 invasion with endogenous and exogenous Fascin1 expression. F) Compound G2 inhibitory activity on control and Fascin1-transfected DLD-1 cells. First two columns represent the difference between percentage of invasion in control (MOCK) and percentage of invasion in treated cells. Second two columns represent the difference between percentage of invasion in Fascin1-transfected DLD-1 and percentage of invasion in compound G2-treated Fascin1-transfected DLD-1. Note that the effect of compound G2 increased when Fascin1 was overexpressed by transfection. Data is shown as mean $\pm \mathrm{SD}$; compared with the control, $* \mathrm{p}=0,049-0,01$. ** $\mathrm{p}=0,001$ 0,009. *** $\mathrm{p}=0,0001-0,0009 . * * * * \mathrm{p}<0,0001$.

\section{Supplementary material}

Supplementary material S1. Detailed description of experimental protocols.

Supplementary material S2. Fascin1 gene expression in colorectal cell lines. mRNA expression levels of Fascin1 was quantified in eight colorectal cell lines using a $\beta$-actin gene expression for data normalizing.

Supplemental material S3. Colorectal cell line viability assay. The effect of migrastatin and compound G2 concentrations in HCT-116 and DLD-1 cell lines is shown. A-B) 24 hours; C-D) 48 hours; E-F) 72 hours incubation. 
Supplementary material S4. Representative images of immunofluorescence assays for $\mu \mathrm{M}$ compound G2; D) $50 \mu \mathrm{M}$ PD98059 (Mek inhibitor); E) $10 \mathrm{ng} / \mathrm{mL}$ Epidermal Growth Factor (EGF, migration stimulator). For actin staining, cells were fixed and stained with anti-actin antibody (1/1000). Images were captured with a LSM 510 META confocal fluorescence microscope with $63 \mathrm{X}$ oil objective. Actin filaments are show in red in these structures. Co-staining with Hoechst-33258 was used to show the cell nuclei. Lamellipodium are more prominent in control and EGF conditions.

Supplementary material S5. Representative images of immunofluorescence assays for fascin1 (green), nucleus (blue) and actin (red) are shown. A) Control condition (DMSO) with siRNA-A transfected HCT-116 cells; B) Control condition (DMSO) with fascin1 silenced HCT-116 cells; C) Fascin1 silenced HCT-116 cells with 10 ng/mL Epidermal Growth Factor (EGF, migration stimulator), D) Fascin1 silenced HCT-116 cells with 50 $\mu$ M PD98059 (Mek inhibitor). Colorectal carcinoma HCT-116 cells were genetically knocked-down for Fascin1 with short interfering siRNA, control HCT-116 was transfected with siRNA-A (MOCK). Cells were fixed with bouin and co-stained with anti-Fascin1 antibody (1/250) and Hoechst-33258. For actin staining, cells were fixed with methanol and stained with anti-actin antibody (1/1000). Images were captured with a LSM 510 META confocal fluorescence microscope with 63X oil objective.

Supplementary material S6. Transwell invasion assay in HCT-116 cell line. A) Invasiveness of cells after treatment in control conditions, $100 \mu \mathrm{M}$ migrastatin and 20 $\mu \mathrm{M}$ compound $\mathrm{G} 2$, respectively. Pictures were taken under an inverted phase contrast microscope. The magnification was $200 \mathrm{X}$ and scale bars $=50 \mu \mathrm{m}$. B) Quantification of the invasive cells D.O. by a spectrophotometer at $\lambda=560 \mathrm{~nm}$. C) Number of invasive 
cells using the Image $\mathrm{J}$ software. Data is shown as mean $\pm \mathrm{SD}$; compared with the control. $* * \mathrm{p}<0.01$

Supplementary material S7. Inhibition of the migration and invasive capacities by compound G2 in transfected cells. A) Percentage of migration. B) Percentage of invasion. Colorectal carcinoma HCT-116 cells were genetically knocked-down for Fascin1 with short interfering siRNA, MOCK control HCT-116 was transfected with siRNA-A. Colorectal carcinoma DLD-1 cells were genetically overexpressed for Fascin1 with Fascin1-GFP vector, MOCK control DLD-1 was transfected with pGFP N3 control vector. Data are representative of two similar experiments Error bars, mean \pm SD of duplicates. $* \mathrm{p}<0.05, * * \mathrm{p}<0.01$ compared to MOCK condition; n.s: nonsignificant.

Supplementary material S8. Myoma organotypic invasion model in HCT-116 cell line. A) Invasion of the cells within myoma discs after treatment in A) control conditions; B) $100 \mu \mathrm{M}$ migrastatin; C) 20 compound G2 and D) $10 \mu \mathrm{M}$ compound G2. Pictures were taken under a phase contrast microscope with $100 \mathrm{X}$ magnification and $100 \mu \mathrm{m}$ scale bars. E) Invasion area and F) Invasion depth of cytokeratin positive cells were quantified with image $J$. Data is shown as mean \pm SD compared with the control. $* * \mathrm{p}<0.01 * * * \mathrm{p}<0.001$

Supplementary material S9. Viability of treated zebrafish larvae in the E3 medium. A) Non injected larvae. B) Additionally, larvae (>20 per condition) were injected with HCT-116 and treatments were added to the E3 medium. C) Larvae were injected with transfected DLD-1 tumoral cell lines and treated.

Supplementary material S10. Metastasis potential. The number of colonies generated was counted at 6 dpi. A) Individual invading DLD-1 cells. B) Both groups of non- 
transfected and Fascin1-tranfected DLD-1 cells showed a decrease in the number of larvae with metastasis. 
Click here to access/download;Figure;Figure 1.tif $\underline{\underline{\imath}}$

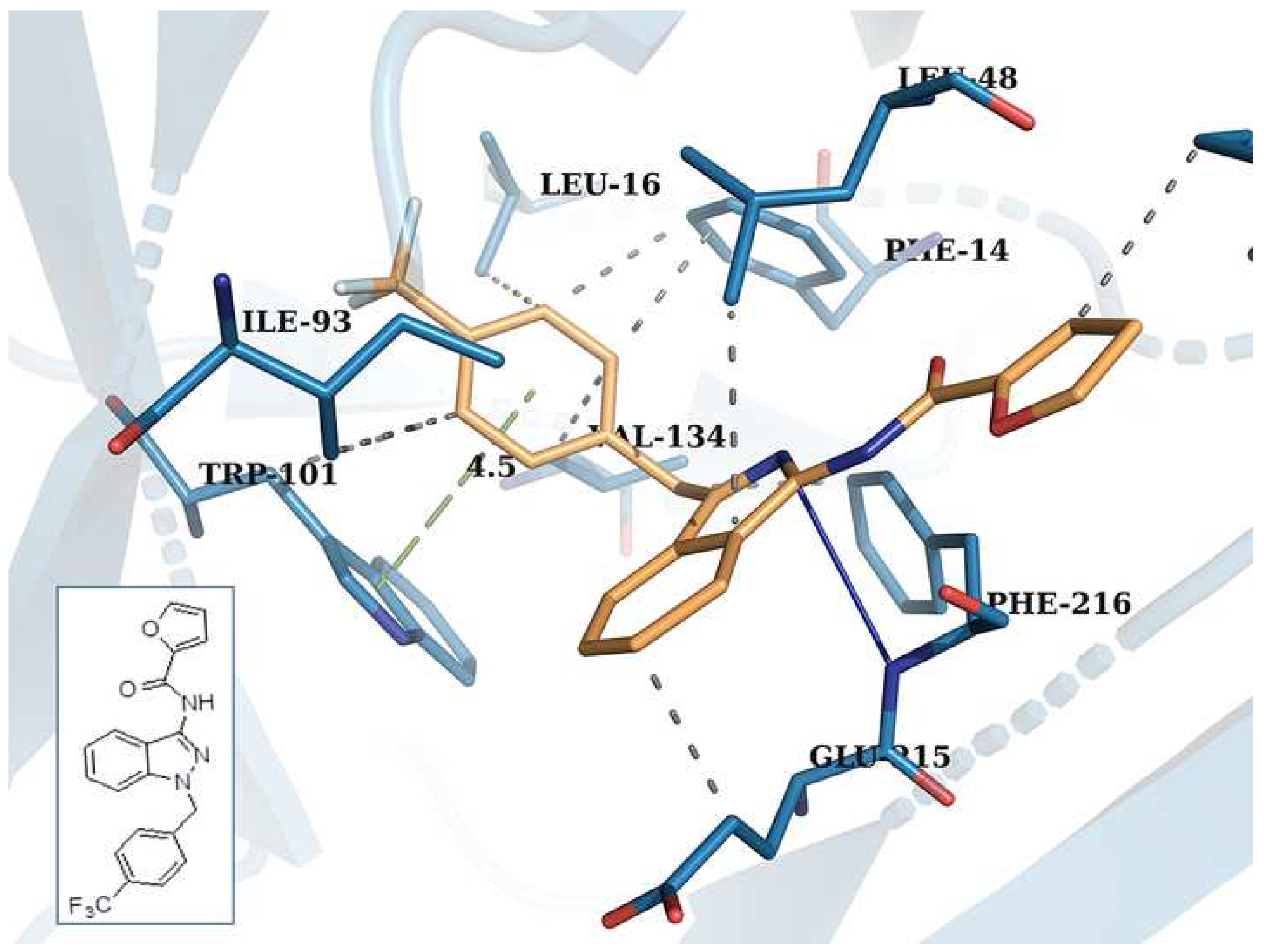




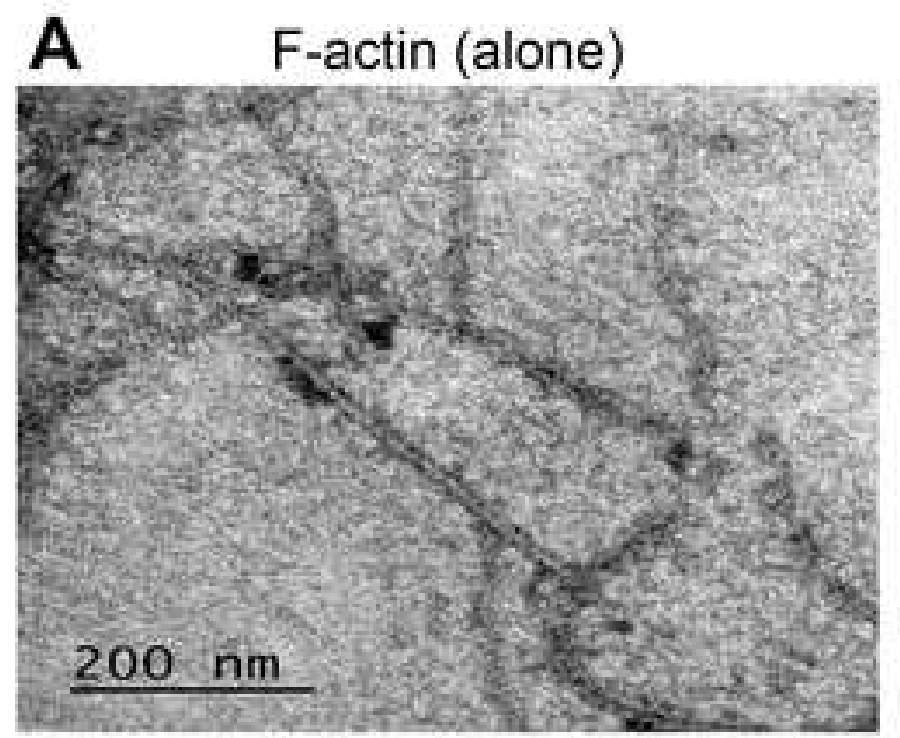

B $\quad$ F-actin + Fascin1 (control)
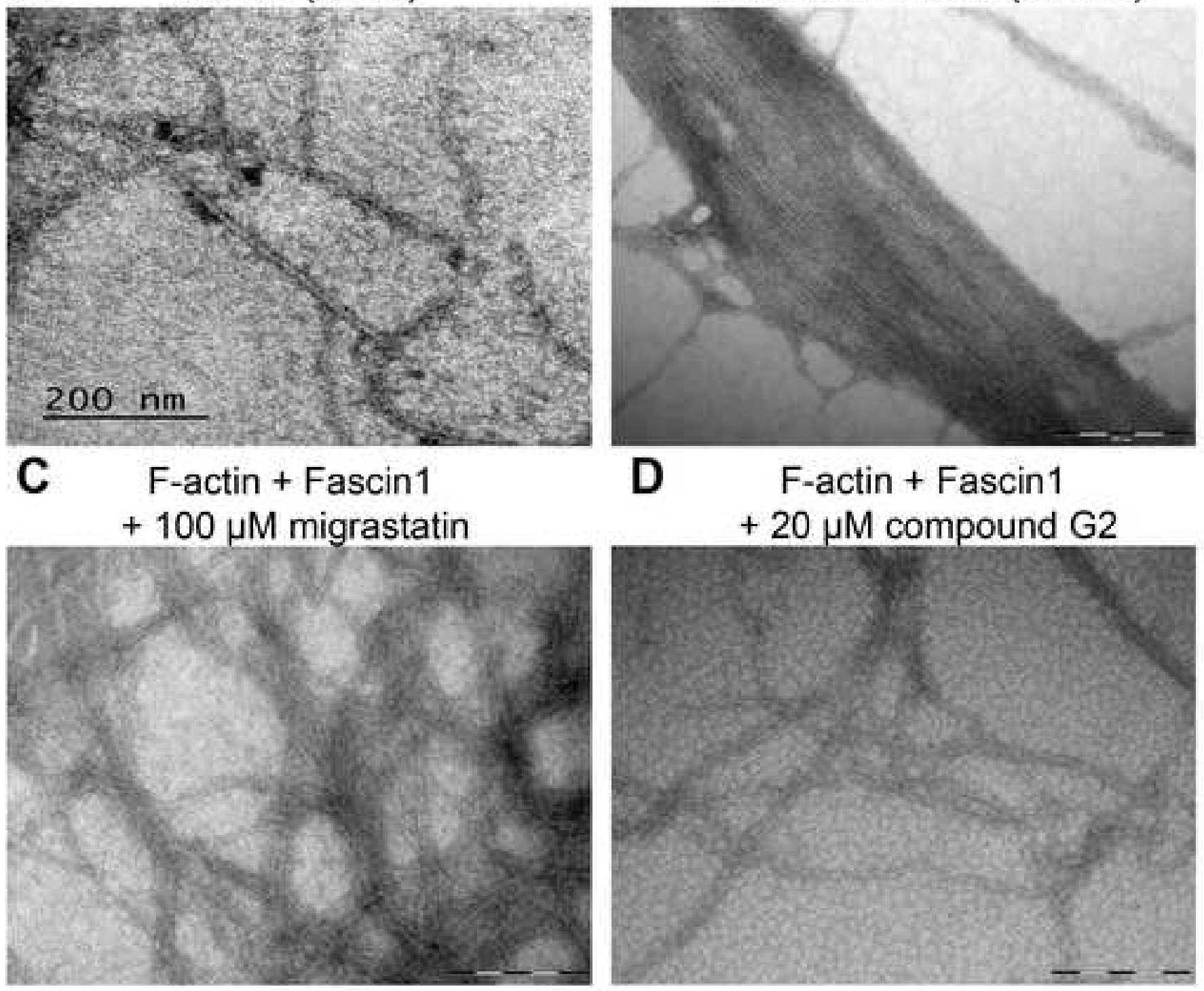

D $\quad \mathrm{F}$-actin + Fascin1 $+20 \mu \mathrm{M}$ compound $\mathrm{G} 2$

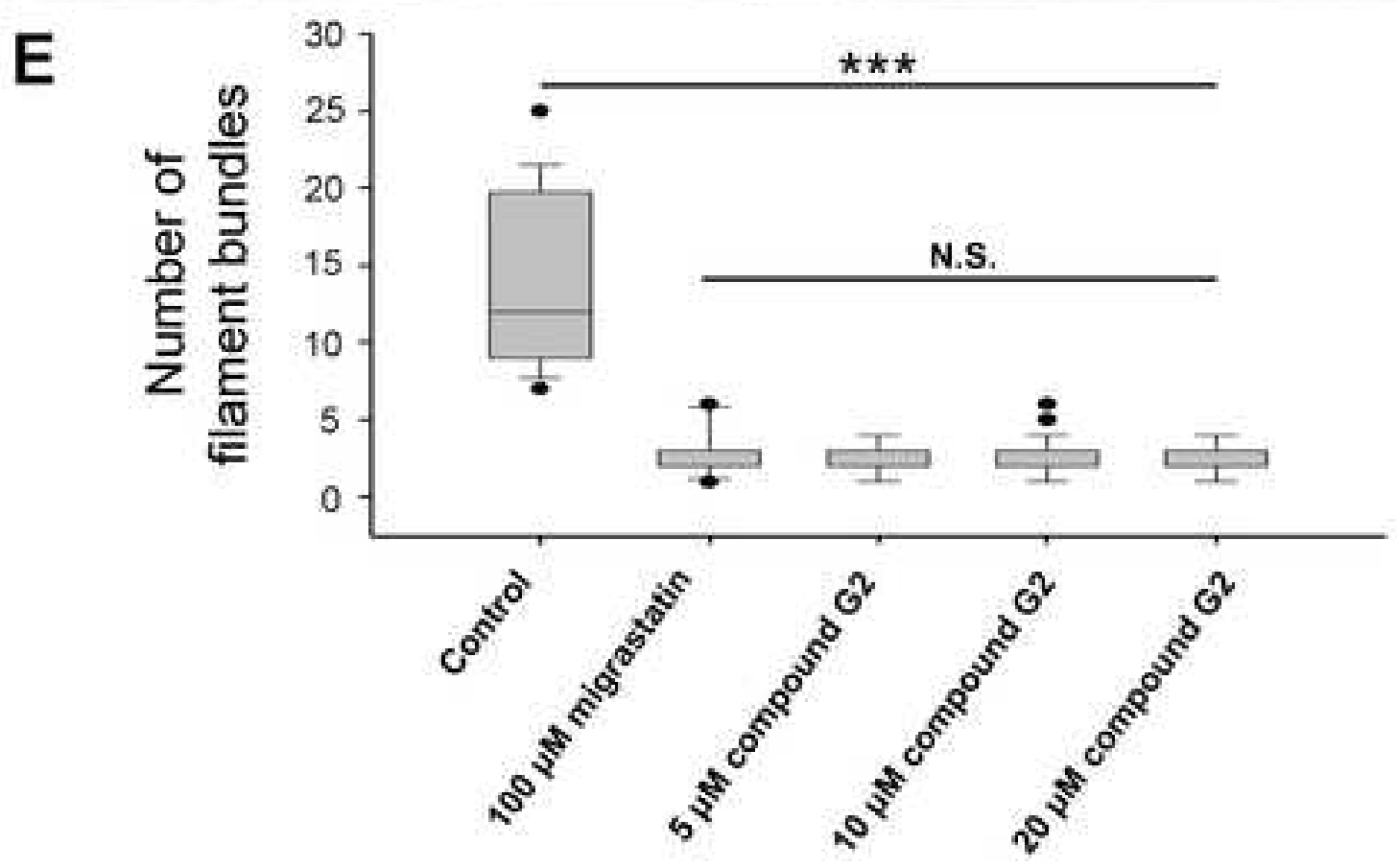



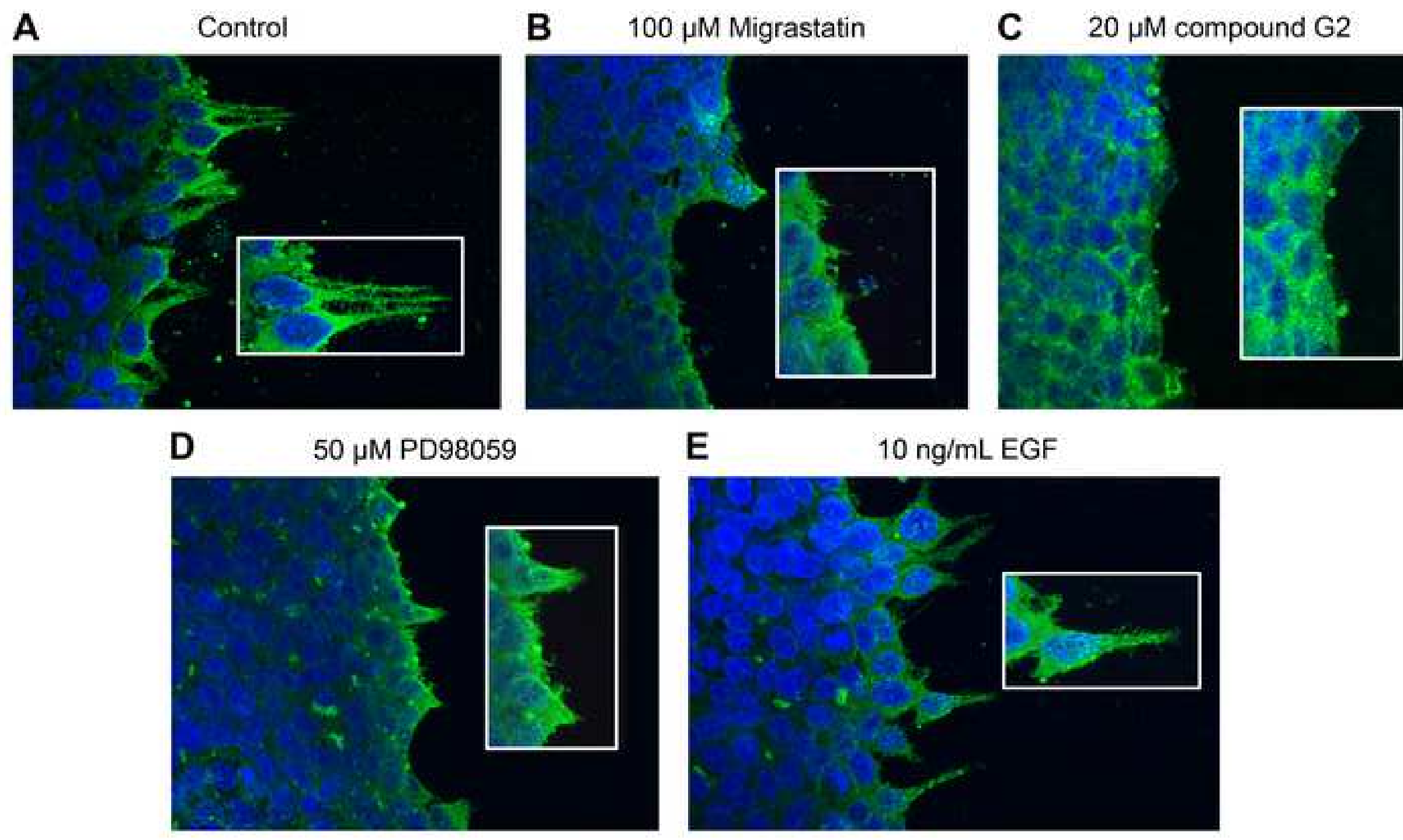


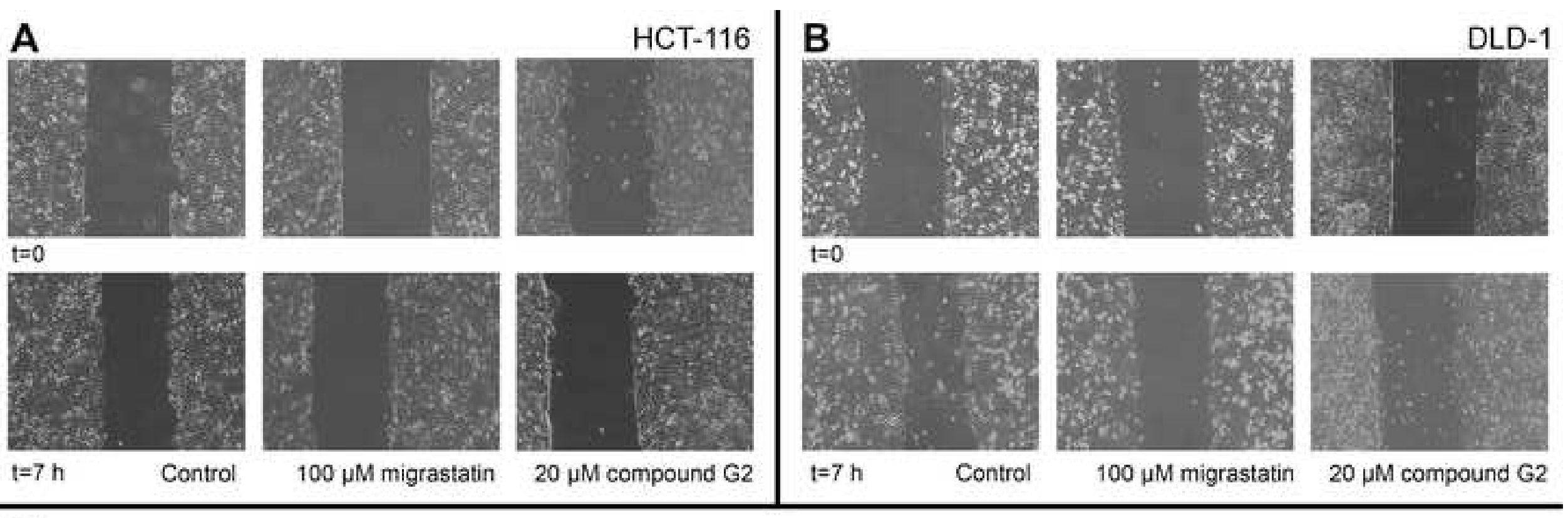

C

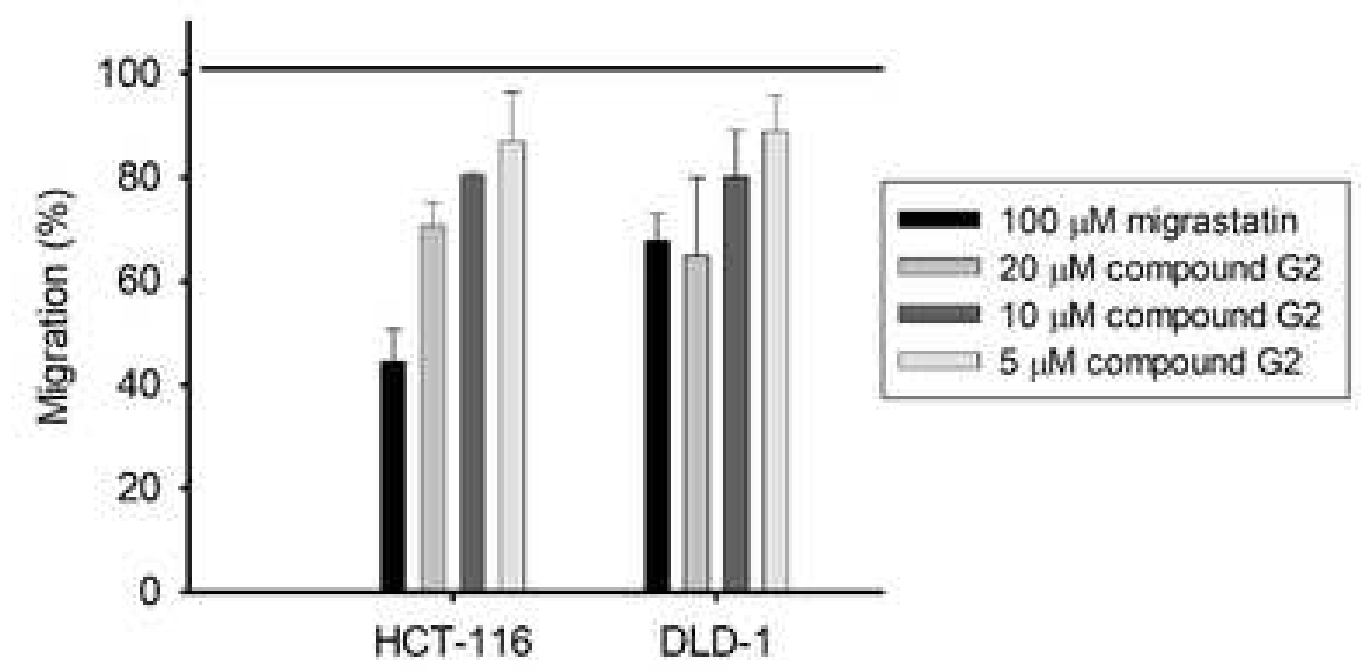




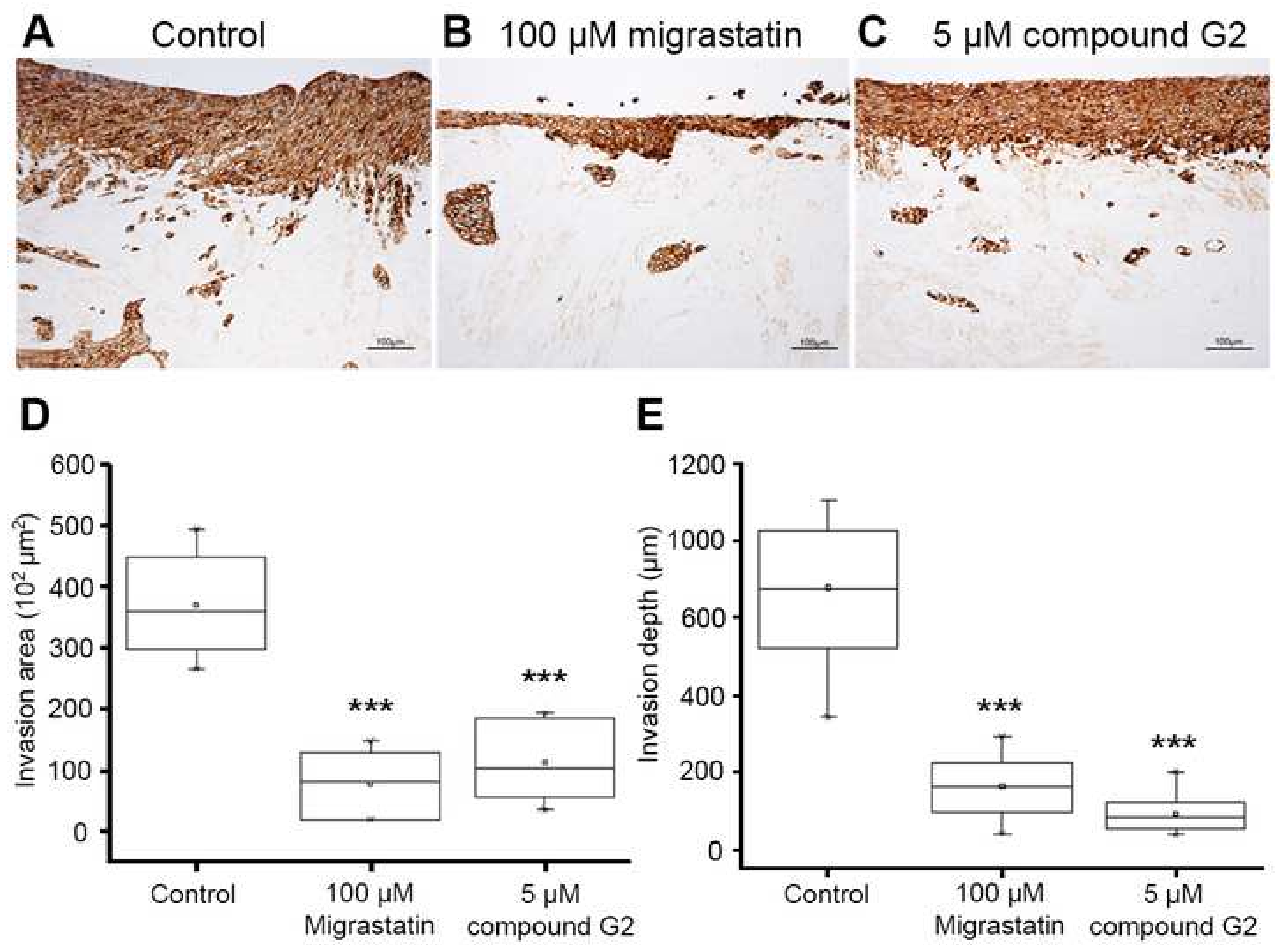

A Control B $100 \mu \mathrm{M}$ migrastatin C $5 \mu \mathrm{M}$ compound G2

D 
A

\section{Non invasive}

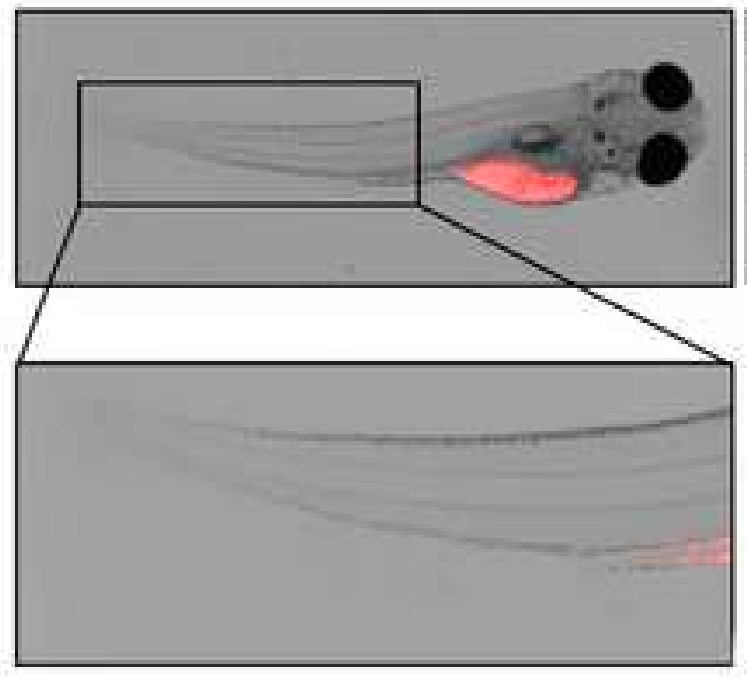

\section{Invasive}

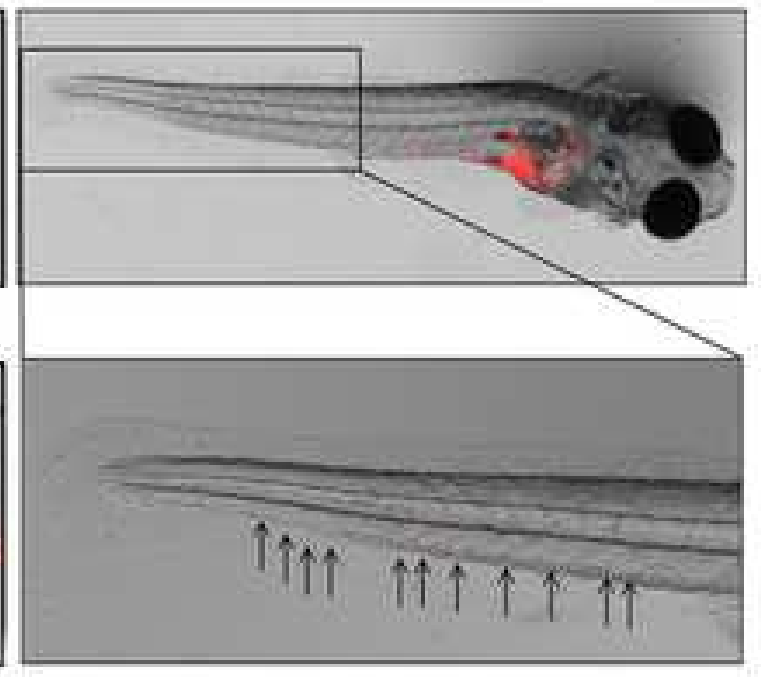

B

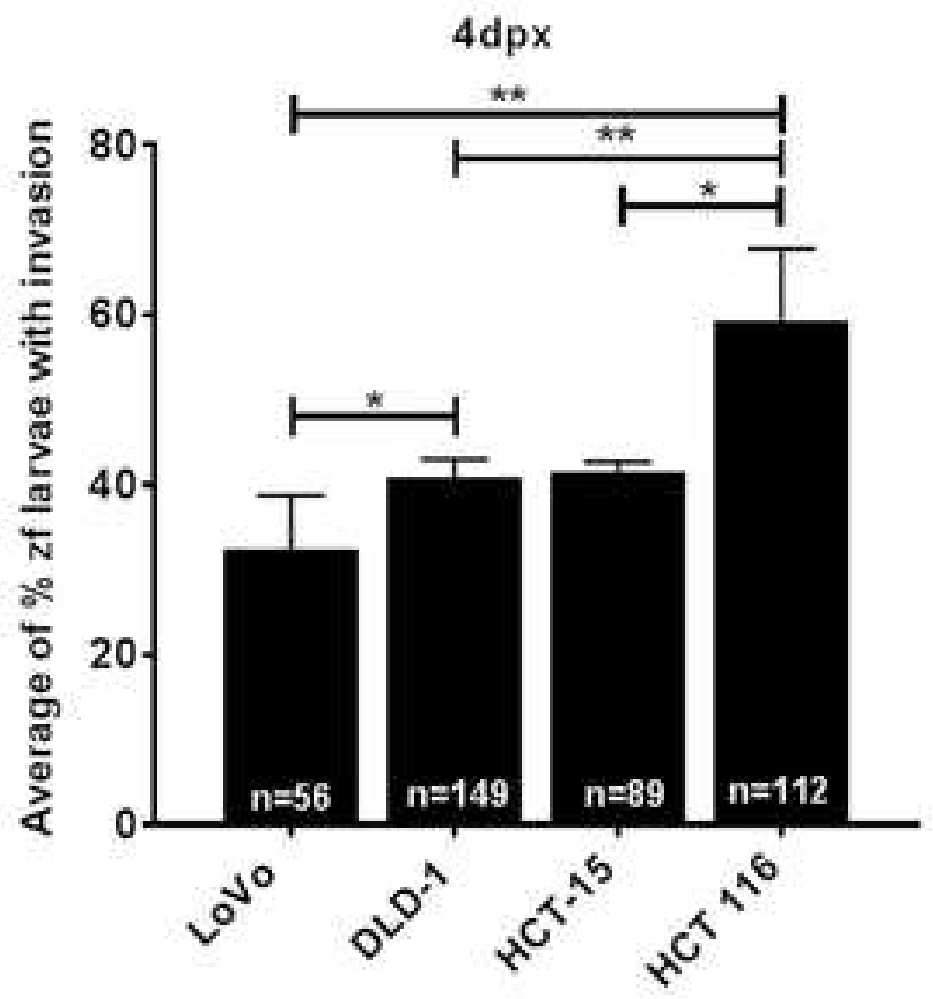


A

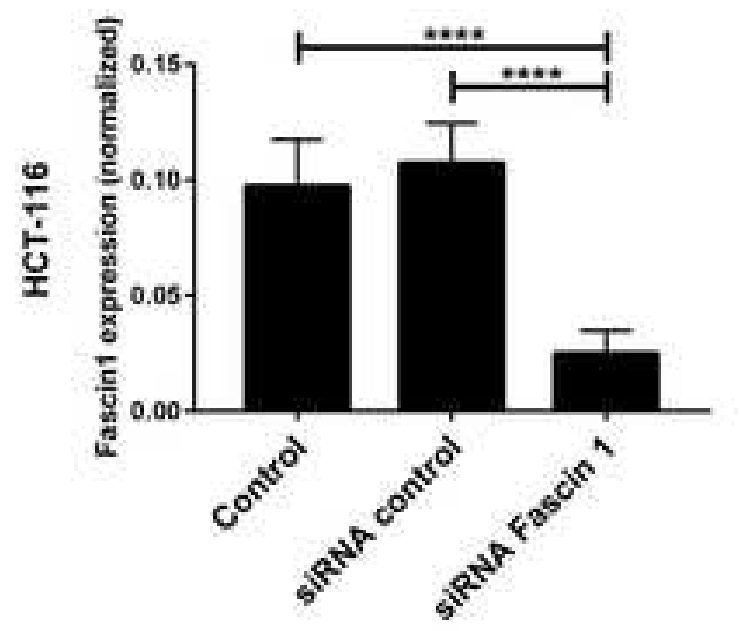

D

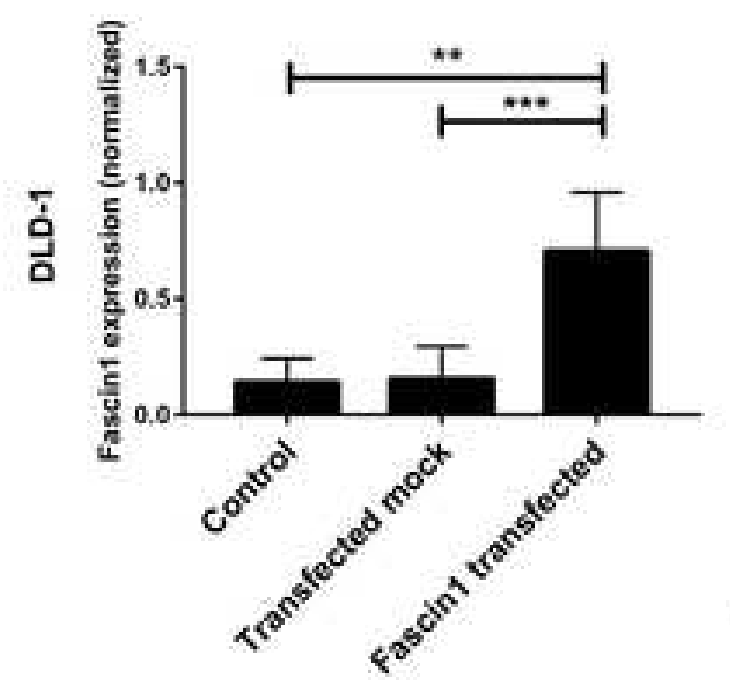

B

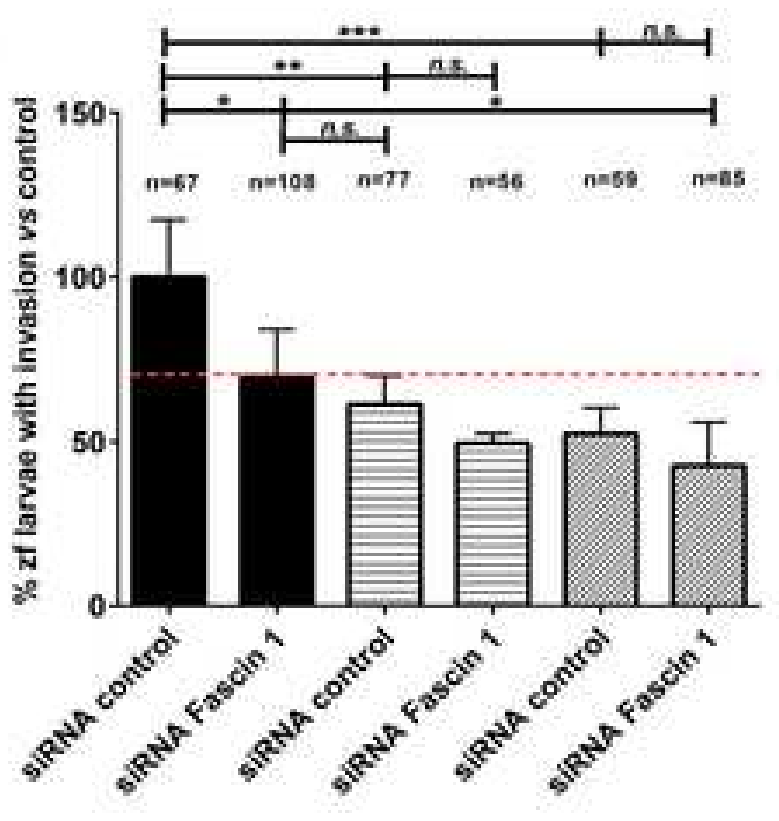

E

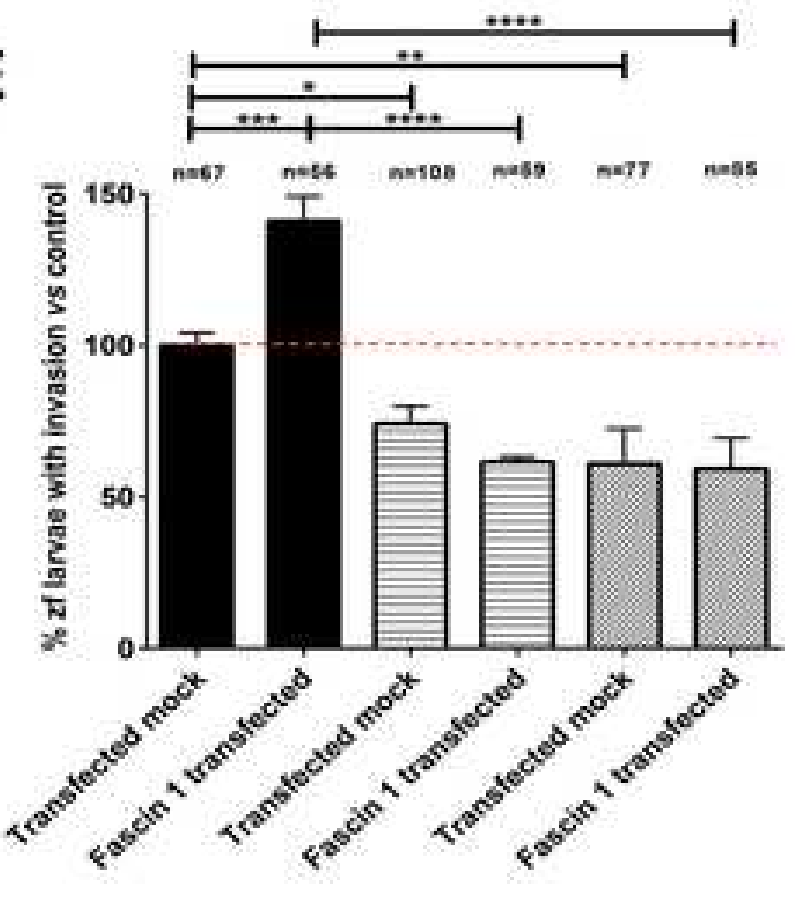

C

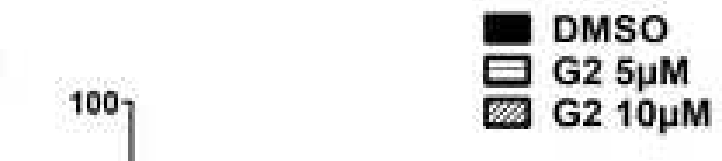

F

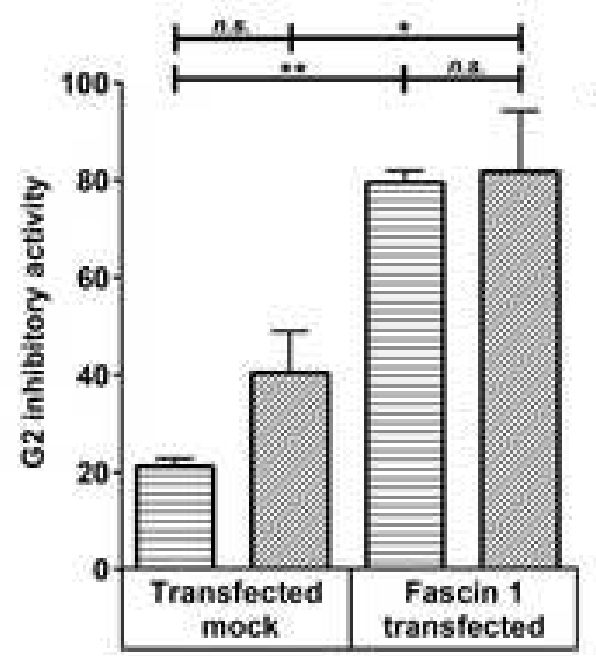


Click here to access/download Supplementary Material Montoro_et_al-Supplementary material R2.docx 